\title{
Holonomy of the Ising model form factors
}

\author{
S. Boukraa ${ }^{\dagger}$, S. Hassani ${ }^{\S}$, J.-M. Maillard ${ }^{\ddagger}$, B. M. McCoy ${ }^{\llbracket}$, \\ W. P. Orrick ${ }^{\star}$ and N. Zenine ${ }^{\S}$ \\ †Université de Blida, LPTHIRM and Département d'Aéronautique, Blida, \\ Algeria \\ $\S$ Centre de Recherche Nucléaire d'Alger, \\ 2 Bd. Frantz Fanon, BP 399, 16000 Alger, Algeria \\ $\ddagger$ LPTMC, Université de Paris 6, Tour 24, 4ème étage, case 121, \\ 4 Place Jussieu, 75252 Paris Cedex 05, France \\ IInstitute for Theoretical Physics, State University of New York, Stony Brook, \\ USA \\ * Department of Mathematics, Indiana University, Bloomington, Indiana 47405, \\ USA \\ E-mail: maillard@lptmc.jussieu.fr, maillard@lptl.jussieu.fr, \\ mccoy@max2.physics.sunysb.edu, boukraa@mail.univ-blida.dz, \\ njzenine@yahoo.com, worrick@indiana.edu
}

\begin{abstract}
We study the Ising model two-point diagonal correlation function $C(N, N)$ by presenting an exponential and form factor expansion in an integral representation which differs from the known expansion of Wu, McCoy, Tracy and Barouch. We extend this expansion, weighting, by powers of a variable $\lambda$, the $j$-particle contributions, $f_{N, N}^{(j)}$. The corresponding $\lambda$ extension of the twopoint diagonal correlation function, $C(N, N ; \lambda)$, is shown, for arbitrary $\lambda$, to be a solution of the sigma form of the Painlevé VI equation introduced by Jimbo and Miwa. Linear differential equations for the form factors $f_{N, N}^{(j)}$ are obtained and shown to have both a "Russian doll" nesting, and a decomposition of the differential operators as a direct sum of operators equivalent to symmetric powers of the differential operator of the elliptic integral $E$. Each $f_{N, N}^{(j)}$ is expressed polynomially in terms of the complete elliptic integrals $E$ and $K$. The scaling limit of these differential operators breaks the direct sum structure but not the "Russian doll" structure. The previous $\lambda$-extensions, $C(N, N ; \lambda)$ are, for singledout values $\lambda=\cos (\pi m / n)$ ( $m, n$ integers), also solutions of linear differential equations. These solutions of Painlevé VI are actually algebraic functions, being associated with modular curves.
\end{abstract}

PACS: 02.30.Hq, 02.30.Gp, 02.30.-f, 02.40.Re, 05.50.+q, 05.10.-a, 04.20.Jb

AMS Classification scheme numbers: 33E17, 33E05, 33Cxx, 33Dxx, 14Exx, 14Hxx, 34M55, 47E05, 34Lxx, 34Mxx, 14Kxx

Keywords: form factors, sigma form of Painlevé VI, two-point correlation functions of the Ising model, Fuchsian linear differential equations, complete elliptic integrals, elliptic representation of Painlevé VI, scaling limit of the Ising model, algebraic solutions of Painlevé VI, modular curves, Eisenstein series, quasi-modular forms. 


\section{Introduction}

The two dimensional Ising model in zero magnetic field is, historically, the most important solvable model in all of theoretical physics. The free energy [1, the partition function on the finite lattice [2] and the spontaneous magnetization [3, 4] were computed long ago by Onsager, Kaufman and Yang. These computations, and subsequent studies of the correlation functions [5]- 9 , form the basis of scaling theory and of the renormalization group approach to critical phenomena.

The next most important macroscopic property of the Ising model, which one would like to compute, is the magnetic susceptibility at zero magnetic field, which is expressed in terms of the two-point correlation functions $C(M, N)$ with a spin at the origin and the other spin in row $M$ and column $N$, as

$$
k_{B} T \cdot \chi=\sum_{M, N=-\infty}^{\infty}\left(C(M, N)-\mathcal{M}^{2}(0)\right)
$$

where $\mathcal{M}(0)$ is the spontaneous magnetization (which is only non zero for $T<T_{c}$ ). Unlike the free energy, and spontaneous magnetization, this has no known closed form expression, and the study of the magnetic susceptibility has been the most challenging outstanding problem in the field for over 50 years.

The first serious analytic study of the susceptibility was made in 1976 by Wu, McCoy, Tracy and Barouch [9] who used their expansions of the correlation functions to write the susceptibility as an infinite series in multiparticle contributions as

$$
\chi_{ \pm}(T)=\sum_{j}^{\infty} \chi^{(j)}
$$

where the subscript \pm refers to $T$ above (resp. below) $T_{c}$ and the sum is over odd (resp. even) values of $j$ for $T$ above (below) $T_{c}$. In ref. 9 the contributions $\chi^{(1)}$ and $\chi^{(2)}$ were explicitly calculated.

No further analysis of the susceptibility $\chi$, or of the $\chi^{(j)}$, was attempted until 1999 when Nickel, in two remarkable papers [10, 11, showed for $j \geq 3$ that the $\chi^{(j)}$ 's have singularities in the complex temperature plane whose number increases with $j$ and become dense on a circle as $j \rightarrow \infty$. Unless a remarkable cancellation takes place this discovery implies that the magnetic susceptibility will have a natural boundary in the complex temperature plane which extends to $T_{c}$. This natural boundary is a new phenomenon which is not incorporated into scaling, or renormalization theory, and, thus, it raises significant questions about our understanding of critical phenomena. Consequently it is most important to deeply understand the properties of the Ising susceptibility, and this challenging question certainely requires some serious progress on the two-point correlation functions of the Ising model. Note that some re-summed high temperature series [12, 13, in the anisotropic case has, already, enabled Guttmann and Enting [13] to conjecture, for the anistropic $\chi$, a natural boundary in one variable when the second variable is fixed.

In 2001 the work of Orrick et al. [14 provided a polynomial time algorithm for obtaining the coefficients of the susceptibility series of the two-dimensional Ising model: from a combinatorial enumerative viewpoint this can be viewed as a "solution" of the problem. The existence of such a polynomial time algorithm for a lattice problem, instead of the exponential growth of the calculations one expects at first sight, can be seen as some "combinatorial integrability" of the model [13. However a (very) efficient way of getting very large series expansions for a physical quantity of 
a model of lattice statistical mechanics, is far from providing the closed formula and exact results one might desire : for instance, there is still a lot of work to be done in order to extract singular points, singular behaviours, from the knowledge of very large series.

In 2004 several of the present authors [15, 16, 17, 18 initiated the study of the Ising susceptibility, beyond the singularity analysis of Nickel [10, 11, by determining the Fuchsian linear differential equations for $\chi^{(3)}$ and $\chi^{(4)}$ as a function of the temperature. These equations have many remarkable properties such as a "Russian doll" nesting structure : the function $\chi^{(1)}$ satisfies the equation for $\chi^{(3)}$ and $\chi^{(2)}$ satisfies the equation for $\chi^{(4)}$. If this nesting can be proven to extend to all of the $\chi^{(j)}$ 's there must be remarkable structures in the Fuchsian equations and the hope is thus raised that it may be possible to characterize the full susceptibility.

In a more recent paper [19] several of the present authors provided new results on the exact expressions of the two-point correlation functions of the Ising model, especially the diagonal correlation $C(N, N)$, underlining the key role played by the second order linear differential operator corresponding to the complete elliptic integral of the first or second kind $K$ or $E$.

In this paper, we study the diagonal correlation functions $C(N, N)$ as a form factors expansion. Our starting point will be the expansions of the diagonal correlations in an exponential form [9], both for $T<T_{c}$

$$
C_{-}(N, N)=(1-t)^{1 / 4} \cdot \exp \left(\sum_{n=1}^{\infty} F_{N, N}^{(2 n)}\right)
$$

with

$$
t=\left(\sinh \left(2 E^{v} / k_{B} T\right) \sinh \left(2 E^{h} / k_{B} T\right)\right)^{-2}
$$

and for $T>T_{c}$

$$
C_{+}(N, N)=(1-t)^{1 / 4} \cdot \sum_{n=0}^{\infty} G_{N, N}^{(2 n+1)} \cdot \exp \left(\sum_{n=1}^{\infty} F_{N+1, N+1}^{(2 n)}\right)
$$

with

$$
t=\left(\left(\sinh \left(2 E^{v} / k_{B} T\right) \sinh \left(2 E^{h} / k_{B} T\right)\right)^{2}\right.
$$

where $E^{h}$ and $E^{v}$ are the horizontal and vertical interaction energies of the Ising model. When the exponentials in (3) and (5) are expanded, the correlations can also be written in what is called a "form factor" expansion :

$$
\begin{aligned}
& C_{-}(N, N)=(1-t)^{1 / 4} \cdot\left(1+\sum_{n=1}^{\infty} f_{N, N}^{(2 n)}\right) \\
& C_{+}(N, N)=(1-t)^{1 / 4} \cdot \sum_{n=0}^{\infty} f_{N, N}^{(2 n+1)}
\end{aligned}
$$

The form factor $f_{N, N}^{(j)}$ is interpreted as the " $j$-particle" contribution to the two-point correlation function. It is natural to consider $\lambda$-extensions [9, 21] of the previous

$\S$ The full susceptibility could be the solution of a nonlinear equation, or the solution of a system of PDE's, or solution of a nonlinear functional equation, or ... 
functions

$$
\begin{aligned}
& C_{-}(N, N ; \lambda)=(1-t)^{1 / 4} \cdot\left(1+\sum_{n=1}^{\infty} \lambda^{2 n} f_{N, N}^{(2 n)}\right) \\
& C_{+}(N, N ; \lambda)=(1-t)^{1 / 4} \cdot \sum_{n=0}^{\infty} \lambda^{2 n} \cdot f_{N, N}^{(2 n+1)}
\end{aligned}
$$

which weight each $f_{N, N}^{(j)}$ by some power of $\lambda$, and to interpret $\lambda$ as being analogous to a coupling constant in a quantum field theory expansion. Such $\lambda$-extensions naturally emerge from the Fredholm determinant framework in [9. We will present new integral representations for $F_{N, N}^{(2 n)}, G_{N, N}^{(2 n+1)}$ and $f_{N, N}^{(j)}$ in sec.(2). We will see that they are much simpler, and more transparent, than the forms obtained from $C(M, N)$ of 9 ] by specializing to $M=N$. The proof of these results is obtained by extending the expansion solution for the leading term given in 1966 by Wu [7, to all orders. It will be published elsewhere.

The diagonal correlations $C(N, N)$ have the property, discovered by Jimbo and Miwa [20] in 1980, that their log-derivatives are solutions of the "sigma" form tit of a Painlevé VI function

$$
\begin{aligned}
& \left(t(t-1) \frac{d^{2} \sigma}{d t^{2}}\right)^{2}=N^{2}\left((t-1) \frac{d \sigma}{d t}-\sigma\right)^{2} \\
& -4 \frac{d \sigma}{d t}\left((t-1) \frac{d \sigma}{d t}-\sigma-\frac{1}{4}\right)\left(t \frac{d \sigma}{d t}-\sigma\right)
\end{aligned}
$$

where $\sigma$ is defined for $T<T_{c}$ as

$$
\sigma_{N}(t)=t(t-1) \cdot \frac{d \ln C_{-}(N, N)}{d t}-\frac{t}{4}
$$

with the normalization condition

$$
C_{-}(N, N)=1+O(t) \text { for } \quad t \rightarrow 0
$$

and, for $T>T_{c}$, as

$$
\sigma_{N}(t)=t(t-1) \cdot \frac{d \ln C_{+}(N, N)}{d t}-\frac{1}{4}
$$

with the normalization condition

$$
C_{+}(N, N)=\frac{(1 / 2)_{N}}{N !} \cdot t^{N / 2} \cdot(1+O(t)) \quad \text { for } \quad t \rightarrow 0
$$

where $(a)_{N}=\Gamma(a+N) / \Gamma(a)$ denotes the Pochhammer symbol.

One can easily verify that (11), the $N$-dependent sigma form of Painlevé VI, is actually covariant by the Kramers-Wannier duality :

$$
\left(t, \sigma, \sigma^{\prime}, \sigma^{\prime \prime}\right) \quad \rightarrow \quad\left(\frac{1}{t}, \frac{\sigma}{t}, \sigma-t \cdot \sigma^{\prime}, t^{3} \cdot \sigma^{\prime \prime}\right)
$$

On another hand, Jimbo and Miwa introduced in 20] an isomonodromic $\lambda$ extension of $C(N, N)$ and showed that this more general function $C(N, N ; \lambda)$ also satisfies (11). The motivation of introducing an isomonodromic parameter $\lambda$, in the framework of isomonodromy deformations, is, at first sight, quite different from the "coupling constant" motivation at the origin of the form factor $\lambda$-extensions (9) and (10). In sec.(3) we show that these two $\lambda$-extensions are actually the same by

$\ddagger$ We use a variable $t$ which is the inverse of the one of Jimbo and Miwa [20]. 
demonstrating that the recursive solutions of (11), analyti_ in $t^{1 / 2}$, agree with (9) and (10) where the $f_{N, N}^{(j)}$ 's are obtained from $C_{ \pm}(N, N ; \lambda)$, the $\lambda$ expansion of $C_{ \pm}(N, N)$ of sec.(2). The normalization condition (13) fixes one integration constant in the solution to (11). We find that the second integration constant is a free parameter, and, denoting that parameter by $\lambda$, that our one parameter family of solutions for $C_{-}(N, N)$ can be written in a form structurally similar to the right hand side of (9). Furthermore, we have confirmed, by comparison with series expansions of the multiple integral formulas for $f_{N, N}^{(j)}$ derived in sec.(2), that this family of solutions is, in fact, identical to $C_{-}(N, N ; \lambda)$ as defined in (9). Similarly, the condition (15) gives rise to a one parameter family of solutions for $C_{+}(N, N)$ that is identical to (10). After all, the fact that these two distinct $\lambda$-extensions of $C_{ \pm}(N, N)$ identify is not altogether surprising, since Jimbo and Miwa's derivation of (11) also starts from a multipleparticle expansion of the correlation functions in terms of free fermion operators. It does not, however, appear to have been observed previously.

In sec.(4) we use formal computer algebra to study the functions $f_{N, N}^{(j)}$. We obtain the Fuchsian linear differential equations satisfied by the $f_{N, N}^{(j)}$ for fixed $j \leq 9$ and arbitrary $N$. We also find the truly remarkable result that the families $f_{N, N}^{(2 j+1)}$ and $f_{N, N}^{(2 j)}$ are each annihilated by linear differential operators which have a nested "Russian doll" structure. Beyond this "Russian doll" structure, each linear differential operator is the direct sum of linear differential operators equivalent四 to symmetric powers of the second order differential operator corresponding to $f_{N, N}^{(1)}$, (or equivalently to the second order differential operator $L_{E}$, corresponding to the complete elliptic integral $E)$. A direct consequence is that the form factors $f_{N, N}^{(2 j+1)}$, and $f_{N, N}^{(2 j)}$ are polynomials in the complete elliptic integrals of the first and second kinds, $K$ and $E$ :

$$
K={ }_{2} F_{1}(1 / 2,1 / 2 ; 1 ; t), \quad E={ }_{2} F_{1}(1 / 2,-1 / 2 ; 1 ; t)
$$

A simple example is $f_{0,0}^{(2)}=K \cdot(K-E) / 2$.

The closed formula we obtain for the differential operators in these nested "Russian doll" structures, enable us to take the scaling limit of these operators. We study this scaling limit in sec.(5) and show that the "Russian doll" structure remains valid. The differential operators in that "scaled" nested Russian doll structure remain equivalent to the symmetric power of a singled out second order differential operator (corresponding to the modified Bessel function). In contrast, in the scaling limit, the direct sum of operators decomposition structure is lost, and we explain why.

The unexpectedly simple expressions for the form factors $f_{N, N}^{(j)}$ of sections (2)(5), and the corresponding remarkable differential structures, may be used to obtain many further results. We display some of these results in sec.(6). Recalling that, when $\lambda=1$, the Ising correlation functions $C(N, N ; 1)$ satisfy Fuchsian differential equations [19] with an order that grows with $N$, it is quite natural to inquire whether there are any other values of $\lambda$ for which $C(N, N ; \lambda)$ will satisfy a Fuchsian linear differential equation. One such family of $\lambda$ is motivated by the work of Cecotti and Vafa [25] on $N=2$ supersymmetric field theories where they encountered $\lambda$ extensions of the Ising correlations in the scaling limit [21] with ( $m$ and $n$ are integers)

$$
\lambda=\cos (\pi m / n)
$$

I The $\lambda$-extensions (9) and (10) are analytic at $t \sim 0$ in $t$ for $T<T_{c}$ and, when $T>T_{c}$, analytic in $t$ for $N$ even, and in $t^{1 / 2}$ for $N$ odd.

$\sharp$ For the equivalence of linear differential operators, see [22, 23, 24]. 
Indeed, we have found that for $n=3, \cdots, 20$, the functions $C(N, N ; \lambda)$ satisfy Fuchsian linear differential equations whose orders, in contrast with those of the $\lambda=1$ equations [19], do not depend on $N$. More importantly, we find that these solutions are actually algebraic functions of $t$, associated with modular curves.

We conclude, in sec.(7), with a discussion about the significance of our results on the factorization of multiple dimensional integrals.

\section{New integral representations for the $f_{N, N}^{(n)}$ 's}

The form factor expressions for $C(M, N)$ of $9,10,11,26,27,14$ are obtained by expanding the exponentials in (3), and (5), in the form given in [9] as multiple integrals and integrating over half the variables. The form of the result depends on whether the even, or odd, variables of [9] are integrated out. For the general anisotropic lattice, one form of this result is given, for arbitrary $M$ and $N$, in [14. When specialized to the isotropic case the result is

$$
f_{M, N}^{(2 j)}=\hat{C}^{2 j}(M, N), \quad f_{M, N}^{(2 j+1)}=\frac{\hat{C}^{2 j+1}(M, N)}{s}
$$

where $s$ denotes $\sinh (2 K)$, and where

$$
\begin{gathered}
\hat{C}^{j}(M, N)=\frac{1}{j !} \int_{-\pi}^{\pi} \frac{d \phi_{1}}{2 \pi} \cdots \int_{-\pi}^{\pi} \frac{d \phi_{j}}{2 \pi}\left(\prod_{n=1}^{j} \frac{1}{\sinh \gamma_{n}}\right) \\
\times\left(\prod_{1 \leq i \leq k \leq j} h_{i k}\right)^{2}\left(\prod_{n=1}^{j} x_{n}\right)^{M} \cos \left(N \sum_{n=1}^{j} \phi_{n}\right)
\end{gathered}
$$

with :

$$
\begin{aligned}
& x_{n}=s+\frac{1}{s}-\cos \phi_{n}-\left(\left(s+\frac{1}{s}-\cos \phi_{n}\right)^{2}-1\right)^{1 / 2}, \\
& \sinh \gamma_{n}=\left(\left(s+\frac{1}{s}-\cos \phi_{n}\right)^{2}-1\right)^{1 / 2} \\
& h_{i k}=\frac{2\left(x_{i} x_{k}\right)^{1 / 2} \sin \left(\left(\phi_{i}-\phi_{k}\right) / 2\right)}{1-x_{i} x_{k}}
\end{aligned}
$$

In this work, we obtain the expressions of $f_{N, N}^{(j)}$ not by setting $M=N$ in the results of [9], but, rather, from the representations of $C(M, N)$ as an $N$-dimensional Toeplitz determinant with elements

$$
\begin{aligned}
a_{m, n}= & a_{m-n}= \\
& \frac{1}{2 \pi} \cdot \int_{0}^{2 \pi} d \theta e^{-i(m-n) \theta}\left(\frac{\left(1-\alpha_{1} e^{i \theta}\right)\left(1-\alpha_{2} e^{-i \theta}\right)}{\left(1-\alpha_{1} e^{-i \theta}\right)\left(1-\alpha_{2} e^{i \theta}\right)}\right)^{1 / 2}
\end{aligned}
$$

with

$$
\alpha_{1}=0 \quad \text { and } \quad \alpha_{2}=s^{-2}
$$

for the diagonal correlation $C(N, N)$, and

$$
\begin{gathered}
\alpha_{1}=\left(\left(1+s^{2}\right)^{1 / 2}-s\right) \cdot\left(\frac{\left(1+s^{2}\right)^{1 / 2}-1}{s}\right) \quad \text { and } \\
\alpha_{2}=\left(\left(1+s^{2}\right)^{1 / 2}-s\right) \cdot\left(\frac{\left(1+s^{2}\right)^{1 / 2}+1}{s}\right)
\end{gathered}
$$


for the row correlation $C(0, N)$. Our method is to follow Wu's paper [7] in the framework of the general theory of Toeplitz determinants.

For $T<T_{c}$, let us first recall (3.15) of Wu's paper 7], which reduces, for the diagonal correlations $C(N, N), \mathrm{t} \mathbf{q}$ :

$$
\begin{aligned}
(1-t)^{-1 / 4} & \cdot C(N, N) \sim \\
1+ & \frac{1}{(2 \pi)^{2}} \int d \xi \cdot \xi^{N} \cdot\left(\left(1-\alpha_{2} \xi\right)\left(1-\alpha_{2} / \xi\right)\right)^{-1 / 2} \\
& \times \int d \xi^{\prime} \cdot \xi^{\prime-N} \cdot\left(\left(1-\alpha_{2} \xi^{\prime}\right)\left(1-\alpha_{2} / \xi^{\prime}\right)\right)^{1 / 2} \frac{1}{\left(\xi^{\prime}-\xi\right)^{2}}
\end{aligned}
$$

Comparing with (7) we see that the second term in (27) is $f_{N, N}^{(2)}=F_{N, N}^{(2)}$.

Performing the change of variables $\xi=z_{1}$ and $\xi^{\prime}=1 / z_{2}$, deforming the contour of integration for both $z_{1}$ and $z_{2}$ (one has to consider only the discontinuity across the branch cut running from 0 to $\alpha_{2}$ ), and rescaling $z_{1}$ and $z_{2}$, in, respectively, $x_{1}=z_{1} / \alpha_{2}$ and $x_{2}=z_{2} / \alpha_{2}$, we obtain :

$$
\begin{aligned}
f_{N, N}^{(2)}(t)= & F_{N, N}^{(2)}(t)=\frac{t^{(N+1)}}{\pi^{2}} \int_{0}^{1} x_{1}^{N} d x_{1} \int_{0}^{1} x_{2}^{N} d x_{2} \\
& \times\left(\frac{x_{1}\left(1-x_{2}\right)\left(1-t x_{2}\right)}{x_{2}\left(1-x_{1}\right)\left(1-t x_{1}\right)}\right)^{1 / 2}\left(1-t x_{1} x_{2}\right)^{-2}
\end{aligned}
$$
of [7]:

Similarly, when $T>T_{c}$, the leading term for $G_{N, N}^{(1)}$ is given by equation (2.29)

$$
f_{N, N}^{(1)}=G_{N, N}^{(1)}=\frac{-1}{2 \pi i} \int_{C} d z \frac{z^{N-1}}{\left(\left(1-t^{1 / 2} z\right)\left(1-t^{1 / 2} z^{-1}\right)\right)^{1 / 2}}
$$

which, after deforming the contour of integration to the branch cut, and scaling $z=t^{1 / 2} x$, becomes

$$
\begin{aligned}
f_{N, N}^{(1)}(t) & =G_{N, N}^{(1)}(t) \\
& =\frac{t^{N / 2}}{\pi} \cdot \int_{0}^{1} x^{N-1 / 2}(1-x)^{-1 / 2}(1-x t)^{-1 / 2} d x \\
& =t^{N / 2} \cdot \frac{(1 / 2)_{N}}{N !} \cdot{ }_{2} F_{1}\left(\frac{1}{2}, N+\frac{1}{2} ; N+1 ; t\right)
\end{aligned}
$$

where ${ }_{2} F_{1}(a, b ; c ; z)$ is the hypergeometric function 30 .

The full expressions for $F_{N, N}^{(2 n)}$ for $T<T_{c}$, and $F_{N+1, N+1}^{(2 n)}$ and $G_{N, N}^{(2 n+1)}$ for $T>T_{c}$, can be obtained by following the iterative procedure based on (2.9)-(2.16) of $[9$ to all orders, just as the full expressions for $F_{M, N}^{(2 n)}$ and $G_{M, N}^{(2 n+1)}$ with $M \neq 0$ of (2.9)-(2.16) of [9] are obtained, in sections 3 and 4 of [9], by following the procedure of Cheng

$\ddagger$ Although in this paper we take the particular values of $\alpha_{1}$ and $\alpha_{2}$ corresponding to $C(N, N)$, our results are also, mutatis mutandis, applicable to the correlations $C(0, N)$ and to the triangular lattice with $\alpha_{1}$ and $\alpha_{2}$ given by 28 .

I To be precise, note that $\mathrm{Wu}$ considered in his paper, the $C(0, N)$ correlations. From the definition (24) of the entries in the Toeplitz determinant one can consider the diagonal correlations $C(N, N)$ with the replacement (25) instead of (26).

$\S$ For $T<T_{c}, \alpha_{2}=t^{1 / 2}<1$. 
and $\mathrm{Wu} 8$ to all order-4. The details of the "iterative procedure" will be presented elsewher $\ddagger$. These are certainly implicit in the paper of Jimbo and Miwa [20, however, we have not been able to find a reference where they are explicitly written out.

When the low temperature expansion of sec. 3 of $\mathrm{Wu}$ [7] is performed to all orders, we find that (94) holds with

$$
\begin{aligned}
F_{N, N}^{(2 n)}= & \frac{(-1)^{n+1}}{n} \frac{1}{(2 \pi)^{n}} \int \prod_{j=1}^{2 n} \frac{z_{j}^{N} d z_{j}}{1-z_{j} z_{j+1}} \\
& \times \prod_{j=1}^{n}\left(\frac{\left(1-\alpha_{2} z_{2 j}\right)\left(1-\alpha_{2} / z_{2 j}\right)}{\left(1-\alpha_{2} z_{2 j-1}\right)\left(1-\alpha_{2} / z_{2 j-1}\right)}\right)^{1 / 2}
\end{aligned}
$$

from which, after deformation of integration contours and rescaling, one obtains, for $T<T_{c}$, the following new integral representation of $F_{N, N}^{(2 n)}(t)$ :

$$
\begin{aligned}
& F_{N, N}^{(2 n)}(t)=\frac{(-1)^{n+1} t^{n(N+1)}}{n \pi^{2 n}} \times \\
& \quad \times \int_{0}^{1} \prod_{j=1}^{2 n} \frac{x_{j}^{N} d x_{j}}{1-t x_{j} x_{j+1}} \cdot \prod_{j=1}^{n}\left(\frac{x_{2 j-1}\left(1-x_{2 j}\right)\left(1-t x_{2 j}\right)}{x_{2 j}\left(1-x_{2 j-1}\right)\left(1-t x_{2 j-1}\right)}\right)^{1 / 2}
\end{aligned}
$$

Similarly for $T>T_{c}$ the expansion of sec.2 of $\mathrm{Wu}$ [7] is performed to all orders and we find that (15) holds with $F_{N, N}^{(2 n)}$ given by (132) and

$$
\begin{aligned}
G_{N, N}^{(2 n+1)} & =(-1)^{n} \frac{1}{(2 \pi)^{2 n+1}} \\
& \times \int \prod_{j=1}^{n+1}\left(z_{j}^{N+1} d z_{j}\right) \frac{1}{z_{1} z_{2 n+1}} \prod_{j=1}^{2 n} \frac{1}{1-z_{j} z_{j+1}} \\
& \times \prod_{j=1}^{n+1}\left(\left(1-\alpha_{2}^{-1} z_{2 j-1}\right)\left(1-\alpha_{2}^{-1} / z_{2 j-1}\right)\right)^{-1 / 2} \\
& \times \prod_{j=1}^{n}\left(\left(1-\alpha_{2}^{-1} z_{2 j}\right)\left(1-\alpha_{2}^{-1} / z_{2 j}\right)\right)^{1 / 2}
\end{aligned}
$$

Changing variables and deforming contours, we obtain :

$$
\begin{aligned}
G_{N, N}^{(2 n+1)}(t) & =(-1)^{n} \frac{t^{N(2 n+1) / 2+2 n}}{\pi^{2 n+1}} \\
& \times \int_{0}^{1} \prod_{j=1}^{2 n+1}\left(x_{j}^{N+1} d x_{j}\right) \frac{1}{x_{1} x_{2 n+1}} \prod_{j=1}^{2 n} \frac{1}{1-t x_{j} x_{j+1}} \\
& \times \prod_{j=1}^{n+1}\left(\frac{x_{2 j-1}}{\left(1-x_{2 j-1}\right)\left(1-t x_{2 j-1}\right)}\right)^{1 / 2}
\end{aligned}
$$

\| The full expressions for $F_{0, N}^{(2 n)}$ and $G_{0, N}^{(2 n-1)}$ can also be obtained by performing the procedure based on (2.9)-(2.16) of [9] to all orders just as the full expressions for $F_{M, N}^{(2 n)}$ and $G_{M, N}^{(2 n-1)}$ with $M \neq 0$ of (2.9)-(2.16) of 9 are obtained in sections 3 and 4 of 9], by "cycling" the procedure of Cheng and $\mathrm{Wu}[8$ to all orders.

$\ddagger$ The first step in that calculation is to consider the ratio $C_{+}(N, N, t) / C_{-}(N, N, t)$. This will be detailed elsewhere. 


$$
\times \prod_{j=1}^{n}\left(\left(1-x_{2 j}\right)\left(1-t x_{2 j}\right) / x_{2 j}\right)^{1 / 2}
$$

The form factor expressions are then obtained by expanding the exponentials. Thus we find, for $T<T_{c}$, that the form factors in (9) read

$$
\begin{aligned}
& f_{N, N}^{(2 n)}(t)=\frac{t^{n(N+n)}}{(n !)^{2}} \frac{1}{\pi^{2 n}} \cdot \int_{0}^{1} \prod_{k=1}^{2 n} x_{k}^{N} d x_{k} \\
& \times \prod_{j=1}^{n}\left(\frac{x_{2 j-1}\left(1-x_{2 j}\right)\left(1-t x_{2 j}\right)}{x_{2 j}\left(1-x_{2 j-1}\right)\left(1-t x_{2 j-1}\right)}\right)^{1 / 2} \\
& \quad \times \prod_{1 \leq j \leq n} \prod_{1 \leq k \leq n}\left(1-t x_{2 j-1} x_{2 k}\right)^{-2} \\
& \quad \times \prod_{1 \leq j<k \leq n}\left(x_{2 j-1}-x_{2 k-1}\right)^{2}\left(x_{2 j}-x_{2 k}\right)^{2}
\end{aligned}
$$

and, for $T>T_{c}$, the odd form factors in (10) read

$$
\begin{aligned}
& f_{N, N}^{(2 n+1)}(t)=t^{((2 n+1) N / 2+n(n+1))} \cdot \frac{1}{\pi^{2 n+1}} \cdot \frac{1}{n !(n+1) !} \\
& \quad \times \int_{0}^{1} \prod_{k=1}^{2 n+1} x_{k}^{N} d x_{k} \prod_{j=1}^{n+1}\left(\left(1-x_{2 j}\right)\left(1-t x_{2 j}\right) x_{2 j}\right)^{1 / 2} \\
& \quad \times \prod_{j=1}^{n+1}\left(\left(1-x_{2 j-1}\right)\left(1-t x_{2 j-1}\right) x_{2 j-1}\right)^{-1 / 2} \\
& \quad \times \prod_{1 \leq j \leq n+1} \prod_{1 \leq k \leq n}\left(1-t x_{2 j-1} x_{2 k}\right)^{-2} \\
& \quad \times \prod_{1 \leq j<k \leq n+1}\left(x_{2 j-1}-x_{2 k-1}\right)^{2} \prod_{1 \leq j<k \leq n}\left(x_{2 j}-x_{2 k}\right)^{2}
\end{aligned}
$$

where the last product in (36) has to be taken to be equal to unity for $n=0,1$. We note that the factors $1 /(n !)^{2}$ and $1 /(n !(n+1) !)$ in (35)), and (36), arise because the integrands are symmetric functions of the variables $x_{2 j}$ and $x_{2 j-1}$, separately. This is to be contrasted with (20) where there is no separation in the odd and even integrals $\phi_{j}$.

In the simplest case the previous integral representation (36) gives $f_{N, N}^{(1)}(t)$ defined by (301) where one recognizes the Euler representation of an hypergeometric function.

Do note that the $\left(G_{N, N}^{(2 n+1)}, F_{N+1, N+1}^{(2 n)}\right)$ decomposition in (5) is not unique. In contrast, the form factor expressions (35), (36) are unique and well-defined.

It is tempting to try to "bridge" such new integral representations (35), (36) with integral formulas like (20), or other integral formulas one can find in 7, , 8, 9, 10, 11, 29, getting (35), (36) from these other integral formulas after some changes of variables, or from partial integrations on a subset of variables in order to reduce $(4 n)$ integral formulas into $(2 n)$ integrals. We have not been able to do this. Basically we have two kinds of drastically different formulas: the ones emerging from Fredholm determinant expansions that naturally yield integral formulas with integrands that are algebraic functions of the self-dual variable $w=s /\left(1+s^{2}\right) / 2$, and the ones emerging from 
"isomonodromic" calculation $\$$ that naturally yield integral formulas with integrands that are algebraic functions of the modulus $k$ of the elliptic function (or the variables $s$ or $t$; for $T>T_{c}, k=s^{2}, t=s^{4}$ ) and break the duality $s \rightarrow 1 / s$. Do note that, in the case of the isotropic lattice, the formulas in [9, 29, are naturally integral formulas with integrands that are algebraic functions of the self-dual variable $w=s /\left(1+s^{2}\right) / 2$.

It is only in the scaling limit that these integral formulas look like integral formulas with integrands that are algebraic functions of $s$, or $k$, or $t$. The " $t$-integral formulas" of the second kind (35), (36) naturally produce series expansions in the hypergeometric functions ${ }_{2} F_{1}$, while the " $w$-integral formulas" of the first kind naturally generate series expansions in the hypergeometric functions ${ }_{4} F_{3}$ : see for instance, all the series calculations we obtained in [15, 16, 17, 18, in the holonomic analysis of $\chi^{(3)}$ and $\chi^{(4)}$. The ${ }_{4} F_{3}$ we consider are particular and, consequently, can be written, for fixed parameters, in terms of ${ }_{2} F_{1}$ for which quadratic transformations $\$$ take place [30]:

$$
\begin{aligned}
& { }_{2} F_{1}\left(a, b ; 2 b ; 16 w^{2}\right)= \\
& \quad\left(1+t^{1 / 2}\right)^{2 a} \cdot{ }_{2} F_{1}(a, a-b+1 / 2 ; b+1 / 2 ; t)
\end{aligned}
$$

We have not been able to prove equality of the two kinds of formulas, so, instead, we have resorted to comparison of their series expansions. We have performed series expansions of our new integral representations in the variable $t$ (see sec.(44), and found that they agree with the expansion of (20). In the next section, we will see that they also agree with the coefficients of powers of $\lambda, h_{2 j}(N, N)(t)$ and $h_{2 j+1}(N, N)(t)$, in the series solutions of (11)-(15).

Our new integral representations provide a "closed enough" representation of the $\lambda$ coefficients of the various $\lambda$ extensions $C(N, N ; \lambda)$, the form factors. We will use the simplicity of these new integral representations in the next sections.

\section{Series solution of the sigma form of Painlevé VI}

In this section we study the series expansions of the diagonal correlations $C(N, N)$ starting from (11), the sigma form of Painlevé VI. From order by order series analysis of the solutions of the equation (11), we show, when $N$ is integer, the existence of a one parameter $\lambda$ extension of $C(N, N)$, that actually identifies with the previous $\lambda$ extension of a Toeplitz origin in [7, or of a Fredholm origin in [9].

We begin by considering some remarkably simple solutions of (11), which exist for all $N$ (not necessarily integer). In particular, consider the $N$-dependent second order hypergeometric differential operator [19]:

$$
L_{h}=D_{t}^{2}+\left(\frac{1}{t}+\frac{1}{2(t-1)}\right) \cdot D_{t}-\frac{1}{4} \frac{N^{2}}{t^{2}}+\frac{1}{16(t-1)^{2}}
$$

It has regular singularities at $t=0, t=1$ and $t=\infty$ with respectively the critical exponents $( \pm N / 2),(1 / 4,1 / 4)$ and $(1 / 4 \pm N / 2)$. Denote by $h$ any solution of (38), and consider the $T>T_{c}$ expression (14) for $\sigma(t)$ :

$$
\sigma(t)=t(t-1) \cdot \frac{d \ln h}{d t}-\frac{1}{4}
$$

$\ddagger$ For instance formulas similar to formulas (13) and (14) of [20].

$\S$ For $a=b=1 / 2$ this is the Landen transformation [30] on the complete elliptic integral $K$. 
The hypergeometric differential equation (38), when written in $\sigma(t)$ given by (39), takes a "Riccati" form :

$$
\begin{aligned}
16 t(t-1) \cdot \sigma^{\prime}+ & 16 \cdot \sigma^{2}-8(t-1) \cdot \sigma \\
& -(2 N-1)(2 N+1)(t-1)^{2}=0
\end{aligned}
$$

For generic $N$, it can be verified that $\sigma(t)$, given by (39), is actually a solution of the sigma form of Painlevé (11), where $h$ is any linear combination of the two solutions of (38) which, for generic $N$, read

$$
f_{ \pm}=t^{ \pm N / 2} \cdot(1-t)^{1 / 4} \cdot{ }_{2} F_{1}(1 / 2,1 / 2 \pm N ; 1 \pm N ; t)
$$

and, for integer $N$, are $f_{+}$and

$$
t^{N / 2} \cdot(1-t)^{1 / 4} \cdot{ }_{2} F_{1}([1 / 2, N+1 / 2],[1], 1-t)
$$

We recognize from (30) that :

$$
f_{+}=(1-t)^{1 / 4} \cdot \frac{N !}{(1 / 2)_{N}} \cdot f_{N, N}^{(1)}
$$

When $t \sim 0$ the leading behavior of $f_{+}$is $f_{+} \sim t^{N / 2}$ which, if we make the normalization

$$
h_{N}=\frac{(1 / 2)_{N}}{N !} \cdot f_{+}=(1-t)^{1 / 4} \cdot f_{N, N}^{(1)}
$$

has the required behavior (15) for the high-temperature two-point correlation function $C_{+}(N, N)$ as $t \sim 0$. When the series expansion of $h_{N}$ is compared with the series expansion of $C_{+}(N, N)$ [19], we find that

$$
\begin{aligned}
C_{+}(N, N)= & (1-t)^{1 / 4} \cdot f_{N, N}^{(1)} \\
+ & \frac{(1 / 2)_{N} \cdot\left((3 / 2)_{N}\right)^{2}}{16 \cdot \Gamma(N+2) \cdot \Gamma(N+3)^{2}} \cdot t^{3 N / 2+2}+\cdots
\end{aligned}
$$

Thus, the first $N+1$ terms of $C_{+}(N, N)$, and $(1-t)^{1 / 4} f_{N, N}^{(1)}$, coincide. The coefficient of $t^{3 N / 2+2}$ can be considered as an "initial condition" needed to complete the characterization of the high-temperature two-point correlation function $C_{+}(N, N)$ seen as a solution of the Painlevé VI equation (11).

For $T<T_{c}$ one notes that

$$
\sigma=t(t-1) \cdot \frac{d \ln \left((1-t)^{1 / 4}\right)}{d t}-\frac{t}{4}
$$

is a trivial solution of (11) and that

$$
C_{-}(N, N)=(1-t)^{1 / 4}+\frac{(1 / 2)_{N} \cdot(3 / 2)_{N}}{4 \cdot((N+1) !)^{2}} \cdot t^{N+1}+\cdots
$$

where the coefficient in front of $t^{N+1}$ is the initial condition defining the two-point correlation function $C_{-}(N, N)$ in the low-temperature regime [19, 31].

The relations (45) and (47) strongly suggest that, in order to analyze solutions of (11), we should introduce the following form for the low temperature expansions

$$
C_{-}(N, N)=(1-t)^{1 / 4} \cdot\left(1+\sum_{k=1}^{\infty} c_{k} \cdot t^{k}\right)
$$

$\ddagger$ Note that, (40), the Riccati form of (38), is also covariant by the Kramers-Wannier duality (16). This is a quite surprising result for a differential equation associated to $f^{(1)}(N, N)$, a form factor one could think to be specific of the $T>T_{c}$ regime. 
and, similarly, for the high temperature expansions :

$$
C_{+}(N, N)=(1-t)^{1 / 4} \cdot f_{N, N}^{(1)}+(1-t)^{1 / 4} \cdot t^{N / 2} \cdot \sum_{k=1}^{\infty} d_{k} \cdot t^{k}
$$

These expansions are not the most general solutions of (11) because we have required that the solutions have the correct behavior (13) and (15) at $t=0$. These forms yield a one parameter family of solutions.

We consider first the low temperature regime and use the form (48) in (11) to determine the $c_{k}$ coefficients recursively, order by order. When $N$ is not an integer this recursive procedure gives the unique solution $c_{k}=0$ for all $k$. Thus the solution $(1-t)^{1 / 4}$ is the only solution of the form (48).

However, when $N$ is an integer, we find that, while $c_{k}=0$ for $k \leq N$, the equation which generically would determine $c_{N+1}$ is automatically satisfied for all values of $c_{N+1}$. The coefficient $c_{N+1}$ can be specified arbitrarily and provides the second "initial" condition needed to specify a unique solution of (11).

For all $k \geq N+2$ the order by order procedure uniquely determines $c_{k}$ as a polynomial in term of the free parameter $c_{N+1}$. More specifically, the term $\left(c_{N+1}\right)^{n}$ first appears in the coefficient $c_{n(N+n)}$. Recalling (47) we see that if one writes the free parameter $c_{N+1}$ as

$$
c_{N+1}=\lambda^{2} \cdot \frac{(1 / 2)_{N} \cdot(3 / 2)_{N}}{4 \cdot((N+1) !)^{2}}
$$

the order by order solution to (11) reads :

$$
C_{-}(N, N ; \lambda)=(1-t)^{1 / 4} \cdot\left(1+\sum_{n=1}^{\infty} \lambda^{2 n} \cdot h_{2 n}(N)\right)
$$

where $h_{2 n}(N) \sim t^{n(N+n)}$ for $t \sim 0$. This $\lambda$ extension $C_{-}(N, N ; \lambda)$ reduces to the low-temperature two-point correlation function $C_{-}(N, N)$ when $\lambda=1$, and to $(1-t)^{1 / 4}$ for $\lambda=0$. Using (51) in (11) we have obtained the low-temperature series expansions of $h_{2 n}(N)$ for a large set of values of the integer $N$, and found that these series expansions actually agree with the series expansions of $(2 n)$-multiple integrals $f_{N, N}^{(2 n)}$ defined in sec. 2 .

A similar order by order expansion can be carried out for the high temperature case. The corresponding coefficients $d_{k}$ can be deduced recursively, order by order. When $N$ is not an integer the recursive procedure gives the unique solution $d_{k}=0$ for all $k$. For non integer $N$ we see that $h_{N}=(1-t)^{1 / 4} \cdot f_{N, N}^{(1)}$ is the unique solution of (11) of the form (49).

However, similar to the case for $T<T_{c}$, we find that, when $N$ is an integer, the coefficients $d_{k}$ are equal to zero for $k \leq N+1$, and that the coefficient $d_{N+2}$ is a free undetermined constant. The coefficients $d_{k}$, for $k \geq N+3$, are polynomials in $d_{N+2}$. The term $\left(d_{N+2}\right)^{n}$ first appears in the coefficient $d_{n(N+n+1)}$. Thus, recalling (45)), we see that if we set

$$
d_{N+2}=\lambda^{2} \cdot \frac{(1 / 2)_{N} \cdot\left((3 / 2)_{N}\right)^{2}}{16 \cdot \Gamma(N+2) \cdot \Gamma(N+3)^{2}}
$$

the iterative solution to (11) may be written, in the high temperature regime, in the form

$$
C_{+}(N, N ; \lambda)=(1-t)^{1 / 4} \cdot \sum_{n=0}^{\infty} \lambda^{2 n} \cdot h_{2 n+1}(N)
$$


where

$$
h_{1}(N)=f_{N, N}^{(1)}
$$

and where $h_{2 n+1}(N) \sim t^{n(N+n+1)}$ for $t \sim 0$. This reduces to the high-temperature two-point correlation function $C_{+}(N, N)$ when $\lambda=1$ and to the hypergeometric function $(1-t)^{1 / 4} \cdot f_{N, N}^{(1)}$ for $\lambda=0$. Using (53) in (11) we have obtained the hightemperature series expansions of $h_{2 j+1}(N)$ 's for a large set of values of the integer $N$, and found that these series expansions agree with $(2 j+1)$-multiple integrals $f_{N, N}^{(2 j+1)}$ defined in sec.(2).

We have also performed low, and high, series expansions for the $\hat{C}^{j}(N, N)$ defined by equations (4.2) in [14] (see also (201)), and we also found that these series identify with the one of $h_{2 j}(N)$ and $h_{2 j+1}(N)$ with the normalization :

$$
h_{2 j}(N)=\hat{C}^{2 j}(N, N), \quad h_{2 j+1}(N)=\frac{\hat{C}^{2 j+1}(N, N)}{s}
$$

It would be most satisfying if these identities could be demonstrated analytically.

All the previous results confirm that these various $\lambda$ extensions identify and actually verify (11). The (log-derivative) of the $\lambda$ extensions $C(N, N ; \lambda)$ satisfy the same (sigma-form of ) Painlevé VI equation (11) as the original diagonal spin-spin correlation, the boundary condition dependence coming from the original diagonal spin-spin correlation boundary condition. Even if some might consider that this result is not mathematically proved, it is clearly an exact result of experimental mathematics, based on an accumulation of large computer formal calculations.

\section{Fuchsian linear differential equations for $f_{N, N}^{(j)}(t)$}

In previous studies on the Ising susceptibility [15, 16, 17, 18, efficient programs were developed which, starting from large series expansions of a holonomic function, produce the linear ordinary differential equation (in this case Fuchsian) satisfied by the function. In order for these programs to be used to study the $f_{N, N}^{(j)}$ 's we need to efficiently produce large (up to several thousand terms) series expansions in $t$ of the $f_{N, N}^{(j)}$ 's. We have done this by use of both the integral representations (35), (36) and the representations of $f_{N, N}^{(j)}$ in terms of theta functions of the nome of elliptic functions, presented in [14].

We obtain the Fuchsian linear differential equations satisfied by the (diagonal) form factors $f_{N, N}^{(j)}$ for $j \leq 9$. The analysis of these linear differential operators shows a remarkable Russian-doll structure similar to the nesting of (the differential operators of) the $\tilde{\chi}^{(j)}$ 's found in [15, 16, 17, 18. Specifically we find that the expressions $f_{N, N}^{(1)}$, $f_{N, N}^{(3)}, f_{N, N}^{(5)}, f_{N, N}^{(7)}$ are actually solutions of the linear ODE for $f_{N, N}^{(9)}$, and that $f_{N, N}^{(0)}$, $f_{N, N}^{(2)}, f_{N, N}^{(4)}, f_{N, N}^{(6)}$ are actually solutions of the ODE for $f_{N, N}^{(8)}$. In addition, we find that all the linear differential operators for the $f_{N, N}^{(j)}$ 's have a direct sum decomposition in operators equivalent to symmetric powers of the differential operator corresponding to $f_{N, N}^{(1)}$. Consequently, all the $f_{N, N}^{(j)}$ 's can also be written as polynomials in terms of the complete elliptic integrals $E$ and $K$. The remainder of this section is devoted to the presentation of these results. 
Holonomy of the Ising model form factors

4.1. Fuchsian linear differential equations for $f_{N, N}^{(2 n+1)}$

The linear differential operator $F_{9}(N)$ which annihilates $f_{N, N}^{(9)}$ has the following factorized form :

$$
F_{9}(N)=L_{10}(N) \cdot L_{8}(N) \cdot L_{6}(N) \cdot L_{4}(N) \cdot L_{2}(N)
$$

where the differential operators $L_{r}(N)$ are of order $r$. The first two read:

$$
\begin{aligned}
& L_{2}(N)=D t^{2}+\frac{2 t-1}{(t-1) t} \cdot D t-\frac{1}{4 t}+\frac{1}{4(t-1)}-\frac{N^{2}}{4 t^{2}} \\
& L_{4}(N)=L_{4,0}-N^{2} \cdot L_{4,2}+\frac{9}{16} \frac{N^{4}}{t^{4}}
\end{aligned}
$$

with:

$$
\begin{aligned}
L_{4,0} & =D t^{4}+10 \frac{(2 t-1)}{(t-1) t} \cdot D t^{3}+\frac{\left(241 t^{2}-241 t+46\right)}{2} \cdot D t^{2} \\
+ & \frac{(2 t-1)\left(122 t^{2}-122 t+9\right)}{(t-1)^{3} t^{3}} \cdot D t+\frac{81}{16} \frac{(5 t-1)(5 t-4)}{t^{3}(t-1)^{3}} \\
L_{4,2} & =\frac{5}{2} \frac{D t^{2}}{t^{2}}-\frac{(23-32 t)}{2(t-1) t^{3}} \cdot D t-\frac{9}{8} \frac{8-17 t}{(t-1) t^{4}}
\end{aligned}
$$

The expressions (or forms) of $L_{6}(N), L_{8}(N)$ and $L_{10}(N)$ are given in Appendix A The linear differential operators $F_{2 n+1}(N)$, which annihilate $f_{N, N}^{(2 n+1)}$ for $n=0, \cdots, 3$, are such that:

$$
\begin{aligned}
& F_{7}(N)=L_{8}(N) \cdot L_{6}(N) \cdot L_{4}(N) \cdot L_{2}(N) \\
& F_{5}(N)=L_{6}(N) \cdot L_{4}(N) \cdot L_{2}(N) \\
& F_{3}(N)=L_{4}(N) \cdot L_{2}(N) \\
& F_{1}(N)=L_{2}(N)
\end{aligned}
$$

Thus we see that the differential operator for $f_{N, N}^{(2 n-1)}$ rightdivides the differential operator for $f_{N, N}^{(2 n+1)}$ for $n \leq 3$. We conjecture that this property holds for all values of $n$. We thus have a "Russian-doll" (telescopic) structure of these successive differential operators.

\subsection{Fuchsian linear differential equations for $f_{N, N}^{(2 n)}$}

The linear differential operator $F_{8}(N)$ (corresponding to $f_{N, N}^{(8)}$ ) has the following factorized form:

$$
F_{8}(N)=L_{9}(N) \cdot L_{7}(N) \cdot L_{5}(N) \cdot L_{3}(N) \cdot L_{1}(N)
$$

where the linear differential operators $L_{r}(N)$ are of order $r$. The first two read:

$$
\begin{aligned}
& L_{1}(N)=D t, \\
& \begin{aligned}
L_{3}(N)=D t^{3}+ & 4 \frac{(2 t-1)}{t(t-1)} \cdot D t^{2}+\frac{\left(2-15 t+14 t^{2}\right)}{(t-1)^{2} t^{2}} \cdot D t \\
& +\frac{8 t^{2}-15 t+5}{2(t-1)^{3} t^{2}}-\left(\frac{D t}{t^{2}}+\frac{1}{t^{3}}\right) \cdot N^{2}
\end{aligned}
\end{aligned}
$$


The expressions (or forms) of the linear differential operators $L_{5}(N), L_{7}(N)$ and $L_{9}(N)$ are given in Appendix A.

Similarly to (61) there is a Russian-doll (telescopic) structure of these successive linear differential operators :

$$
\begin{aligned}
& F_{6}(N)=L_{7}(N) \cdot L_{5}(N) \cdot L_{3}(N) \cdot L_{1}(N) \\
& F_{4}(N)=L_{5}(N) \cdot L_{3}(N) \cdot L_{1}(N) \\
& F_{2}(N)=L_{3}(N) \cdot L_{1}(N) \\
& F_{0}(N)=L_{1}(N)
\end{aligned}
$$

Again, we see that the linear differential operator for $f_{N, N}^{(2 n-2)}$ rightdivides the linear differential operator for $f_{N, N}^{(2 n)}$ for $n \leq 4$. We conjecture that this property holds for all values of $n$.

\subsection{Direct sum structure}

Not only do the linear differential operators $L_{j}(N)$ have a factorized Russian doll structure, but we have found that they also have a direct sum decomposition when the integer $N$ is fixed. To illustrate this direct sum decomposition, the corresponding linear differential operator for $f_{N, N}^{(3)}$ reads:

$$
F_{3}(N)=L_{4}(N) \cdot L_{2}(N)=M_{4}(N) \oplus L_{2}(N)
$$

where $L_{2}(N)$ is the linear differential operator for $f_{N, N}^{(1)}$ and the fourth order operator $M_{4}(N)$ is displayed in Appendix B for successive values of $N$. One remarks on these successive expressions that the degree of each polynomial occurring in these linear differential operators $M_{4}(N)$ grows linearly with $N$.

As a further example consider $f^{(5)}(N, N)$, where we find that the corresponding linear differential operator decomposes as:

$$
F_{5}=L_{6}(N) \cdot L_{4}(N) \cdot L_{2}(N)=M_{6}(N) \oplus M_{4}(N) \oplus L_{2}(N)
$$

where $L_{2}(N)$ is the differential operator for $f_{N, N}^{(1)}, M_{4}(N)$ is the previous fourth order differential operator, and the sixth order operator $M_{6}(N)$ has again coefficients whose degrees grow with $N$ for successive values of $N$. There is nothing specific to $f_{N, N}^{(3)}$ and $f_{N, N}^{(5)}$ : similar results hold for all the $f_{N, N}^{(n)}$ 's, $n$ being even or odd.

In contrast with the Russian-doll way of writing the differential operators for $f_{N, N}^{(n)}$, the direct sum structure, as a consequence of this growing degree, cannot be written for generic $N$ as operators with polynomials in front of the derivatives. This "non-closure" of the direct sum structure will have some consequences when performing the scaling limit of these differential operators (see sec.(5) below).

\subsection{Equivalence of various $L_{j}(N)$ 's and $M_{j}(N)$ 's linear differential operators}

We find that the symmetric square of $L_{2}(N)$ :

$$
\operatorname{Sym}^{2}\left(L_{2}(N)\right)=D t^{3}+3 \frac{(2 t-1)}{(t-1) t} \cdot D t^{2}+\frac{\left(1-7 t+7 t^{2}\right)}{(t-1)^{2} t^{2}} \cdot D t
$$

I The symmetric $j$-th power of a second order linear differential operator having two solutions $f_{1}$ and $f_{2}$ is the linear differential operator of order $j+1$, which has $f_{1}^{j}, \ldots, f_{1}^{j-k} f_{2}^{k}, \ldots, f_{2}^{j}$ as solutions. 
Holonomy of the Ising model form factors

$$
-\frac{1}{2} \frac{1-2 t}{(t-1)^{2} t^{2}}-\frac{N^{2}}{t} \cdot D t-\frac{N^{2}}{(t-1) t^{2}}
$$

and the linear differential operator $L_{3}(N)$ are equivalent :

$$
L_{3}(N) \cdot U(N)=V(N) \cdot \operatorname{Sym}^{2}\left(L_{2}(N)\right)
$$

with the following intertwinners :

$$
\begin{gathered}
U(N)=(t-1) t \cdot D t^{2}+(3 t-1) \cdot D t+1+\frac{(1-t)}{t} \cdot N^{2} \\
V(N)=(t-1) t \cdot D t^{2}+(11 t-5) \cdot D t \\
\quad+\frac{(5 t-1)(5 t-4)}{(t-1) t}-\frac{(t-1)}{t} \cdot N^{2}
\end{gathered}
$$

Similarly, with the symmetric cube of $L_{2}(N)$, we have the equivalence

$$
L_{4}(N) \cdot A(N)=B(N) \cdot \operatorname{Sym}^{3}\left(L_{2}(N)\right)
$$

with :

$$
\begin{aligned}
A(N) & =(t-1) t \cdot D t^{3}+\frac{7}{2}(2 t-1) \cdot D t^{2}+\frac{\left(41 t^{2}-41 t+6\right)}{4(t-1) t} \cdot D t \\
& +\frac{9}{8} \frac{2 t-1}{(t-1) t}-\frac{9}{4} \frac{(t-1) \cdot N^{2}}{t} \cdot D t-\frac{9}{8} \frac{(2 t-1)}{t^{2}} \cdot N^{2} \\
B(N) & =(t-1) t \cdot D t^{3}+\frac{23}{2}(2 t-1) \cdot D t^{2} \\
+ & \frac{21}{4} \frac{\left(6-29 t+29 t^{2}\right)}{(t-1) t} \cdot D t+\frac{9}{8} \frac{(2 t-1)\left(125 t^{2}-125 t+16\right)}{(t-1)^{2} t^{2}} \\
& -\frac{9}{4} \frac{(t-1)}{t} \cdot N^{2} \cdot D t-\frac{9}{8} \frac{(10 t-9)}{t^{2}} \cdot N^{2}
\end{aligned}
$$

More generally, all the $L_{m}(N)$ 's are $(m-1)$-symmetric-power of $L_{2}(N)$. As a consequence their solutions are $(m-1)$-homogeneous polynomial of the two hypergeometric solutions of $L_{2}(N)$.

Similarly, for the linear differential operators occurring in the direct sum, one easily verifies, for every integer $N$, that, for instance, the $M_{4}(N)$ 's are equivalent to the cubic-symmetric-power of $L_{2}(N)$ :

$$
M_{4}(N) \cdot Q(N)=S(N) \cdot \operatorname{Sym}^{3}\left(L_{2}(N)\right)
$$

where, for $N=0,1,2$ :

$$
\begin{aligned}
& Q(0)=(t-1) t \cdot D t+t-\frac{1}{2}, \\
& \begin{array}{l}
Q(1)=2(t-1)^{3} t^{2} \cdot D t^{3}+3\left(3-7 t+4 t^{2}\right)(t-1) t \cdot D t^{2} \\
+\left(12 t^{3}-28 t^{2}+\frac{41}{2} t-\frac{9}{2}\right) \cdot D t+\frac{3}{4} \frac{2 t^{2}-2 t+1}{t}, \\
Q(2)=\frac{1}{3}(t-1)^{3}\left(3+8 t+3 t^{2}\right) t \cdot D t^{3} \\
+\frac{1}{2}\left(15-t-35 t^{2}+15 t^{3}+6 t^{4}\right)(t-1) \cdot D t^{2} \\
-\frac{1}{24} \frac{\left(18 t^{5}-12 t^{4}-97 t^{3}+577 t^{2}-738 t+252\right)}{t} \cdot D t \\
-\frac{1}{16} \frac{12 t^{5}+14 t^{4}-260 t^{3}+497 t^{2}-314 t+24}{t^{2}}
\end{array}
\end{aligned}
$$


As a further example, one can verify, for every value of the integer $N$, that the sixth order operator $M_{6}(N)$ is equivalent to the fifth symmetric power of $L_{2}(N)$. The solutions of the linear differential operators $M_{m}(N)$ are also $(m-1)$-homogeneous polynomials of the two hypergeometric solutions of $L_{2}(N)$.

As a consequence of this direct sum decomposition, the solutions $f^{(n)}(N, N)$ are (non-homogeneous) polynomials of the two hypergeometric solutions of $L_{2}(N)$ or, equivalently, $f_{N, N}^{(1)}$ (or $h_{N}$ see (44)) and its first derivative. The second order linear differential operator $L_{2}(N)$ is equivalent [19] to the second order differential operator $L_{E}$

$$
L_{E}=4 t \cdot D t^{2}+4 D t-\frac{1}{t-1}
$$

corresponding to the complete elliptic integral $E$. As a consequence of the previously described direct sum decomposition, the $f_{N, N}^{(n)}$ 's can also be written as polynomial expressions of the complete elliptic integral $E$ and its first derivative $E^{\prime}$, or alternatively, $E$ and the complete elliptic integral $K$.

Let us just give here a set of miscellaneous examples. For $f_{N, N}^{(2)}$, one has:

$$
\begin{aligned}
& 2 f_{0,0}^{(2)}=(K-E) \cdot K \\
& 2 f_{1,1}^{(2)}=1-3 K E-(t-2) \cdot K^{2} \\
& 6 t \cdot f_{2,2}^{(2)}=6 t-\left(2+6 t^{2}-11 t\right) \cdot K^{2} \\
& \quad \quad-(15 t-4) \cdot K E-2(1+t) \cdot E^{2} \\
& 90 t^{2} f_{3,3}^{(2)}=135 t^{2}-\left(137 t^{3}-242 t^{2}+52 t+8\right) \cdot K^{2} \\
& \quad+\left(8 t^{3}-319 t^{2}+112 t+16\right) \cdot K E-4(1+t)\left(2 t^{2}+13 t+2\right) E^{2} \\
& 3150 t^{3} \cdot f_{4,4}^{(2)}=6300 t^{3} \\
& \quad-\left(32 t^{5}+2552 t^{2}+128+6440 t^{4}-11191 t^{3}+464 t\right) \cdot K^{2} \\
& \quad+\left(128 t^{5}+5648 t^{2}-14519 t^{3}+1056 t+576 t^{4}+256\right) \cdot E K \\
& \quad-8(1+t)\left(16 t^{4}+58 t^{3}+333 t^{2}+58 t+16\right) \cdot E^{2}
\end{aligned}
$$

where $E$ and $K$ are given by (17). Other examples are given in Appendix C.

Remark: All these remarkable structures are not restricted to diagonal two points correlation functions. Actually one can calculate various $j$-particle contributions $f_{M, N}^{(j)}$ of the off-diagonal two point correlation functions, and verify, again, that they are also polynomial expressions of the complete elliptic integrals $E$ and $K$. For instance for $T>T_{c}$ :

$$
\begin{aligned}
C^{(2)}(0,1)= & \frac{3}{8}-\frac{1}{4}\left(1+s^{2}\right) K \\
& -\frac{1}{2} E K-\frac{1}{8}\left(s^{2}-3\right)\left(1+s^{2}\right) \cdot K^{2}
\end{aligned}
$$

where $s=\sinh (2 K)$. Other miscellaneous examples of such off-diagonal $j$-particle contributions are displayed in Appendix D.

\subsection{The elliptic representation of Painlevé VI}

The results we have underlined in this section, namely the unexpectedly simple and remarkable polynomial expressions for the form factors $f_{N, N}^{(j)}$, correspond to the 
fact that the associated linear differential operators are direct sums of operators equivalent to symmetric powers of the second order differential operator $L_{E}$. We already encountered this central key role played by the linear differential operator $L_{E}$, or the hypergeometric second order differential operator (38), in our previous holonomic analysis of the two-point correlation functions of the Ising model [19]. In order to understand the key role played by $L_{E}$, or equivalently operator (38), it is worth recalling (see [32, or for a review [33]) the so-called "elliptic representation" of Painlevé VI. This elliptic representation of Painlevé VI amounts to seeing Painlevé VI as a "deformation" (see equation (33) in [33]) of the hypergeometric linear differential equation associated with the linear differential operator :

$$
\mathcal{L}=(1-t) t \cdot D t^{2}+(1-2 t) \cdot D t-\frac{1}{4}
$$

One easily verifies that this linear differential operator is actually equivalent (in the sense of the equivalence of differential operators) with $L_{E}$, or equivalently (38). This deep relation between elliptic curves and Painlevé VI explains the occurrence of Painlevé VI on the Ising model, and on other lattice Yang-Baxter integrable models which are canonically parametrized in term of elliptic functions (like the eight-vertex Baxter model, the RSOS models, see for instance [34]). We will see, in sec.(6), another example of this deep connection between the transcendent solutions of Painlevé VI and the theory of elliptic functions, modular curves and quasi-modular functions.

\section{The scaling of $f_{N, N}^{(j)}$}

The scaling of the $f_{N, N}^{(n)}$ 's amounts, on the functions, and on the corresponding differential operators, to taking the limit $N \rightarrow \infty$ and $t \rightarrow 1$, keeping the limit $x=N \cdot(1-t)$ finite, or in other words, to performing the change of variables $t=1-x / N$, keeping only the leading term in $N$. Performing these straightforward calculations, the linear differential operators in $t$ for the $f_{N, N}^{(n)}$ 's where $N$ was a parameter, become linear differential operators in the only scaling variable $x$.

Calling $F_{j}^{\text {scal }}$ the scaling limit of the operator $F_{j}(N)$ we find for $j$ even that

$$
\begin{aligned}
& F_{8}^{\text {scal }}=L_{9}^{\text {scal }} \cdot L_{7}^{\text {scal }} \cdot L_{5}^{\text {scal }} \cdot L_{3}^{\text {scal }} \cdot L_{1}^{\text {scal }} \\
& F_{6}^{\text {scal }}=L_{7}^{\text {scal }} \cdot L_{5}^{\text {scal }} \cdot L_{3}^{\text {scal }} \cdot L_{1}^{\text {scal }} \\
& F_{4}^{\text {scal }}=L_{5}^{\text {scal }} \cdot L_{3}^{\text {scal }} \cdot L_{1}^{\text {scal }} \\
& F_{2}^{\text {scal }}=L_{3}^{\text {scal }} \cdot L_{1}^{\text {scal }} \\
& F_{0}^{\text {scal }}=L_{1}^{\text {scal }}
\end{aligned}
$$

where :

$$
\begin{aligned}
& L_{5}^{\text {scal }}=2 x^{5} D x^{5}+10 x^{4} D x^{4}-2 x^{3}\left(7+5 x^{2}\right) D x^{3} \\
& \quad+2\left(-16+13 x^{2}\right) x^{2} D x^{2}+2\left(5-12 x^{2}+4 x^{4}\right) x D x \\
& \quad-10+8 x^{2}-24 x^{4}, \\
& L_{3}^{\text {scal }}=2 x^{3} D x^{3}+8 x^{2} D x^{2}-2(x-1)(x+1) x D x-2, \\
& L_{1}^{\text {scal }}=D x
\end{aligned}
$$

and $L_{9}^{\text {scal }}, L_{7}^{\text {scal }}$ are given in Appendix E. 
Similarly, for $j$ odd, we have

$$
\begin{aligned}
& F_{9}^{\text {scal }}=L_{10}^{\text {scal }} \cdot L_{8}^{\text {scal }} \cdot L_{6}^{\text {scal }} \cdot L_{4}^{\text {scal }} \cdot L_{2}^{\text {scal }} \\
& F_{7}^{\text {scal }}=L_{8}^{\text {scal }} \cdot L_{6}^{\text {scal }} \cdot L_{4}^{\text {scal }} \cdot L_{2}^{\text {scal }} \\
& F_{5}^{\text {scal }}=L_{6}^{\text {scal }} \cdot L_{4}^{\text {scal }} \cdot L_{2}^{\text {scal }} \\
& F_{3}^{\text {scal }}=L_{4}^{\text {scal }} \cdot L_{2}^{\text {scal }} \\
& F_{1}^{\text {scal }}=L_{2}^{\text {scal }}
\end{aligned}
$$

where

$$
\begin{aligned}
L_{4}^{\text {scal }}= & 16 x^{4} D x^{4}+96 x^{3} D x^{3}+40\left(2-x^{2}\right) x^{2} D x^{2} \\
& +8\left(x^{2}-2\right) x D x+9 x^{4}-8 x^{2}+16, \\
L_{2}^{\text {scal }}= & 4 x D x^{2}+4 D x-x
\end{aligned}
$$

and $L_{10}^{\text {scal }}, L_{8}^{\text {scal }}, L_{6}^{\text {scal }}$ are given in Appendix E.

Thus, we see that the scaled operators $F_{j}^{\text {scal }}$ have a "Russian doll" structure inherited from the lattice operators $F_{j}(N)$.

Consider the linear differential operator corresponding to the modified Bessel function $\operatorname{Bessel}(n, x / 2)$ for $n=0$, namely

$$
B=D x^{2}+\frac{D x}{x}-\frac{1}{4}
$$

We recognize, in this linear differential operator, the exact identification with the scaled differential operator $F_{1}^{\text {scal }}=L_{2}^{\text {scal }}$. We find that the symmetric square of the linear differential operator $B$, and the scaled operator $L_{3}^{\text {scal }}$ are equivalent :

$$
\begin{aligned}
L_{3}^{\text {scal }} \cdot( & \left(x D x^{2}+2 D x-x\right)= \\
& \left(2 x^{4} D x^{2}+12 x^{3} D x-2 x^{4}+8 x^{2}\right) \cdot \operatorname{Sym}^{2}(B)
\end{aligned}
$$

Similarly, the symmetric third power of the linear differential operator $B$, and the scaled operator $L_{4}^{\text {scal }}$ are equivalent, and, more generally, the symmetric $j$-th power of (87) and the scaled operator $L_{j+1}^{\text {scal }}$ are equivalent:

$$
L_{j+1}^{s c a l} \simeq \operatorname{Sym}^{j}(B)
$$

Recall that the differential operators $F_{j}(N)$, corresponding to the form factors $f_{N, N}^{(j)}$, can be written as direct sums only when the integer $N$ is fixed. At the scaling limit, this feature dissappears in the scaled differential operators $F_{j}^{\text {scal }}$ which have no direct sums. Therefore while the scaling limit preserves the Russian-doll (telescopic) structure (see (61), (85)) and also preserves the fact that the various operators in this Russian-doll (telescopic) structure are equivalent to symmetric powers of an operator (87) which replaces the operator $L_{E}$, the direct sum structure is lost. As a consequence the scaling of the $f_{N, N}^{(j)}$ 's cannot be seen as simple polynomials of modified Bessel functions.

There is one exception that concerns $f_{N, N}^{(2)}$. Its scaled linear differential operator $F_{2}^{s c a l}$, has the non shared property of being equivalent to the direct sum of $D x$ with the symmetric square of (87), namely:

$$
F_{2}^{s c a l}=L_{1}^{\text {scal }} \oplus L_{3}^{\text {scal }} \simeq D x \oplus \operatorname{Sym}^{2}(B)
$$

From this equivalence, one immediately deduces the expression of the scaling of the $f_{N, N}^{(2)}$ as quadratic expression of the modified Bessel functions of $x / 2$ which actually identifies with formula (2.31b)-(3.151) in [9]. 
The occurrence of modified Bessel functions, emerging from a confluence of two singularities of the complete elliptic integrals $E$ and $K$, or from the hypergeometric function ${ }_{2} F_{1}$, should not be considered as a surprise if one recalls the following limit of the hypergeometric function ${ }_{2} F_{1}$ yielding confluent hypergeometric functions ${ }_{1} F_{1}$. These confluent hypergeometric functions, ${ }_{1} F_{1}$, are nothing but modified Bessel functions [30]:

$$
\begin{aligned}
& { }_{1} F_{1}(a, b ; z) \rightarrow{ }_{2} F_{1}\left(a, p, b ; \frac{z}{p}\right) \quad \text { when: } p \rightarrow \infty \\
& I(\nu, z)=\frac{z^{\nu}}{2^{\nu} e^{z} \Gamma(\nu+1)} \cdot{ }_{1} F_{1}\left(\nu+\frac{1}{2}, 2 \nu+1 ; 2 z\right)
\end{aligned}
$$

Remark: It was shown, in sec.(4), as a consequence of the decomposition of their differential operators in direct sums of operators equivalent to symmetric powers of $L_{E}$, that the functions $f_{N, N}^{(n)}$ are polynomial expressions of $E$ and $K$ functions. Therefore their singularities are only the three regular points $t=0, t=1$ and $t=\infty$. The scaling limit $(t=1-x / N, t \rightarrow 1, N \rightarrow \infty)$ corresponds to the confluence of the two regular points $t=0$ and $t=\infty$, yielding the, now, irregular point $x=\infty$. The occurrence of irregular points with their Stokes phenomenon, and, especially, the loss of a remarkable direct sum structure, shows that the scaling limit is a quite non-trivial limit.

Contrary to the common wisdom, the scaling limit does not correspond to more "fundamental" symmetries and structures (more universal ...) : this limit actually destroys the remarkable structures and symmetries of the lattice model $\$$.

\section{Algebraic solutions of PVI for $\lambda=\cos (\pi m / n)$ and modular curves}

The function $C(N, N ; \lambda)$ is such that its log-derivative is actually a solution of the sigma form of Painlevé VI : it is a transcendent function "par excellence". However, the unexpectedly simple expressions for these form factors $f_{N, N}^{(j)}$, strongly suggest to

try to resum the infinite sums (9), and (10), of form factors $f_{N, N}^{(j)}$, corresponding to the function $C(N, N ; \lambda)$, and see if these transcendent functions could be "less complex" than one can imagine at first sight, at least for a set of "singled-out" values of $\lambda$. For instance, are there any values of $\lambda \neq 1$ which share, with $\lambda=1$, the property that $C(N, N ; \lambda)$ satisfies a Fuchsian linear differential equation?

Actually, introducing, instead of the modulus $k$ of elliptic functions (for $T>T_{c}$, $k=s^{2}$ ), or the $s$ and $t$ variables, the nome of the elliptic functions (see relations (5.7)-(5.11) in [14]), we have been able to perform such a resummation, getting, for arbitrary $\lambda$, nice closed expressions for the $C(N, N ; \lambda)$ for the first values of $N,(N=0,1,2 \cdots)$, as sums of ratios of theta functions (and their derivatives), corresponding to Eisenstein series, or quasi-modular forms. These results will be displayed in forthcoming publications. The simplest example corresponds to $N=0$ where $C_{-}(N, N ; \lambda)$ is just the ratio of two Jacobi $\theta_{3}$ functions :

$$
C_{-}(0,0 ; \lambda)=\frac{\theta_{3}(u, q)}{\theta_{3}(0, q)}, \quad \text { where: } \quad \lambda=\cos (u)
$$

$\S$ These kind of results should not be a surprise for the people working on integrable lattice models, or on Painlevé equations 35, 36. 
All these results strongly suggest to focus on $u=\pi m / n$ ( $m$ and $n$ integers) yielding for the possible choice of "singled-out" values of $\lambda$ :

$$
\lambda=\cos (\pi m / n)
$$

Actually these special values (93) of $\lambda$ already occurred in a study of $N=2$ supersymmetric field theories [25] in a similar series construction of solutions of the Painlevé V (or Painlevé III for a ratio of functions) equation for the scaling limit of the Ising model [9].

We have begun to investigate this situation. When $n=3, \cdots, 20$ (and all the possible values of $m$, but a set of first successive values of $N$ ), we have found that $C_{ \pm}(N, N ; \lambda)$ do indeed satisfy Fuchsian linear differential equations but, unlike the equations found in [19] for $\lambda=1$, the order of the Fuchsian linear differential equations depends only on $n$ and not on $N$.

As examples of these Fuchsian linear differential equations, we found, for instance, that $C_{-}(N, N ; \cos (\pi / 4))$, for $N=0,1,2$, are annihilated, respectively, by

$$
\begin{aligned}
L_{0}^{[1 / 4]}= & (t-1)^{2} t \cdot D t^{2}+\frac{3}{8}(t-1)(3 t-2) \cdot D t-\frac{15 t}{256}+\frac{3}{32} \\
L_{1}^{[1 / 4]}= & (t-1)^{2} t \cdot D t^{2}+(t-1)(5 t-2) \cdot D t-\frac{7 t}{256}+\frac{1}{16} \\
L_{2}^{[1 / 4]}= & (t-8)(t-1)^{2} t \cdot D t^{2}+(t-1)\left(t^{2}-2 t+16\right) \cdot D t \\
& \quad+\frac{209 t^{2}}{256}-\frac{25 t}{16}+\frac{1}{2}
\end{aligned}
$$

and that $C_{-}(0,0 ; \cos (\pi / 3))$ is annihilated by :

$$
\begin{aligned}
L_{0}^{[1 / 3]} & =(t-1)^{3} t^{3} \cdot D t^{4}+\frac{11}{3}(2 t-1)(t-1)^{2} t^{2} \cdot D t^{3} \\
& +\frac{7}{27}\left(43 t^{2}-43 t+4\right)(t-1) t \cdot D t^{2} \\
& +\frac{7}{1458}(2 t-1)\left(247 t^{2}-247 t-80\right) \cdot D t+\frac{35}{486}
\end{aligned}
$$

These linear differential operators are of a quite different nature from the one depicted in sec.(4) which can be decomposed in direct sums of (operators equivalent to) symmetric powers of $L_{E}$. In contrast with the direct sum decomposition we have underlined previously, these linear differential operators are irreducible. However we do expect from sec.(4.5) a connection with elliptic curves. Actually, instead of a connection through the second order differential operator $L_{E}$, or the hypergeometric second order linear differential operator (38), we have an even more striking link with the theory of elliptic curves. These solutions $C(N, N ; \lambda)$ are actually algebraic solutions of Painlevé VI, associated with modular curve $\$$. We found for $n \leq 8$ these singled-out Fuchsian linear differential equations, corresponding to algebraic solutions of Painlevé VI, and beyond, directly these modular curves for larger values of $n$ for which we do not have the Fuchsian linear differential equations yet.

We first obtained these modular curves as polynomial relations $P(\sigma, t)=0$, between $\sigma$ and $t$, and we then found, in a second step, the polynomial relations $P(\tau, t)=0$, between $\tau=C_{ \pm}(N, N ; \cos (\pi m / n))$ and $t$. For instance, one finds that $\tau=C_{-}(0,0 ; \cos (\pi / 3))$ is solution of a genus one algebraic curve :

$$
16 \tau^{12}-16 \tau^{9}-8(t-1) t \cdot \tau^{3}+t \cdot(1-t)=0
$$

$\S$ The occurrence of modular curves is pretty clear for $N=0$ from (92), from the analysis of its invariance group, subgroup of the modular group. 
or that $\tau=C_{-}(N, N ; \cos (\pi / 4))$ is solution of genus three algebraic curve, for instance, for $N=0$,

$$
16 \tau^{16}+16(t-1) \cdot \tau^{8}+t^{2} \cdot(t-1)=0
$$

the corresponding solutions being quite simple algebraic expressions :

$$
\begin{aligned}
C_{-}(0,0 ; \cos (\pi / 4)) & =2^{-1 / 4}(1-t)^{1 / 16}\left[1+(1-t)^{1 / 2}\right]^{1 / 4} \\
C_{-}(1,1 ; \cos (\pi / 4)) & =2^{-3 / 4}(1-t)^{1 / 16}\left[1+(1-t)^{1 / 2}\right]^{3 / 4} \\
C_{-}(2,2 ; \cos (\pi / 4)) & = \\
\quad=2^{-5 / 4} & (1-t)^{1 / 16}\left[1+(1-t)^{1 / 2}\right]^{5 / 4}\left[5-(1-t)^{1 / 2}\right] / 4
\end{aligned}
$$

We give in Table 1, when available, the order of the Fuchsian linear differential equation for $\lambda=\cos (\pi / n)$, the degree and genus of the corresponding algebraic curve $P\left(C_{-}(0,0 ; \lambda), t\right)=0$, and the degree and genus of the algebraic $\sigma$-curve $P(\sigma(0,0 ; \lambda), t)=0$.

\begin{tabular}{|l|l|l|l|l|l|l|l|l|l|l|l|l|l|l|}
\hline$n$ & 3 & 4 & 5 & 6 & 7 & 8 & 9 & 10 & 11 & 12 & 14 & 16 & 18 & 20 \\
\hline ODE order & 4 & 2 & 12 & 4 & 24 & 8 & & & & & & & & \\
\hline$\tau$-degree & 12 & 16 & 60 & 48 & 168 & 128 & & 240 & & & & & & \\
\hline$\tau$-genus & 1 & 3 & 13 & 13 & & 41 & & & & & & & & \\
\hline$\sigma$-degree & 4 & 2 & 12 & 4 & 24 & 8 & 36 & 12 & 60 & 16 & 24 & 32 & 36 & 48 \\
\hline$\sigma$-genus & 0 & 0 & 1 & 0 & 4 & 0 & & 1 & & 1 & & & & \\
\hline
\end{tabular}

Table 1. Order of the linear ODE, as well as degree and genus of the corresponding modular curve in $\tau=C_{ \pm}(0,0 ; \lambda)$ for $\lambda=\cos (\pi / n)$, when available. The corresponding degree and genus of the modular curve in $\sigma(0,0 ; \lambda)$, when available.

We found the following results on the polynomial relations $P(\tau, t)=0$, between $\tau=C_{-}(N, N ; \cos (\pi m / n))$ and $t$. These polynomials are actually polynomials of the variable $\rho=\tau^{n}$ for $n$ odd and of the variable $\rho=\tau^{2 n}$ for $n$ even. This property is related to the invariance of the variable $\rho$ under a subgroup of the modular grour $\boldsymbol{~}$. Let us denote $Q(\rho, t)=0$ the polynomial relation between $\rho$ and $t$. We also found that the degree of the polynomial $Q$ in $\rho$ actually identifies with the degree in $\sigma$ of the polynomial $P(\sigma, t)=0$. Thus, the $\tau$-degree in Table 1 can be seen to be the $\sigma$-degree multiplied by $n$ for $n$ odd, and by $2 n$ for $n$ even. The order of the Fuchsian linear differential equations for $C_{-}(N, N ; \lambda)$ identifies with that degree in $\sigma$. We finally found that the genus of the modular curve $P(\sigma, t)=0$ identifies with the genus of the $\tau^{n}$ (resp. $\tau^{2 n}$ )-modular curve $Q(\rho, t)=0$ : the genus corresponding to $C_{-}(0,0 ; \cos (\pi / 3))^{3}, C_{-}(0,0 ; \cos (\pi / 5))^{5}$ are respectively 0,1 , the genus corresponding to $C_{-}(0,0 ; \cos (\pi / 4))^{8}, \quad C_{-}(0,0 ; \cos (\pi / 6))^{12}, \quad C_{-}(0,0 ; \cos (\pi / 8))^{16}$ are 0 but the genus for $C_{-}(0,0 ; \cos (\pi / 10))^{20}$ is 1 . In contrast the genus corresponding to $C_{-}(0,0 ; \cos (\pi / 6))^{6}, C_{-}(0,0 ; \cos (\pi / 8))^{8}$ are 1 , and the genus for $C_{-}(0,0 ; \cos (\pi / 10))^{10}$ is 5 .

For $N=0$, and only in this case, a large set of these algebraic curves (for instance (96) or the modular curve for $n=7$ in the previous table) are invariant

9 See in particular Barth and Michel [37] for further details on the $X_{00}(n, 2)$ modular curves and the characterization of the genus of modular curves from subgroups of $S L(2, Z)$. We will study $C_{ \pm}(N, N ; \lambda)$ from this modular subgroup point of view elsewhere. 
under the $t \leftrightarrow 1-t$ symmetry :

$$
\left(t, \sigma, \sigma^{\prime}, \sigma^{\prime \prime}\right) \quad \rightarrow \quad\left(1-t,-\sigma-1 / 4, \sigma^{\prime},-\sigma^{\prime \prime}\right)
$$

This remarkable symmetry is, in fact, inherited from the covariance by (101) of the sigma form (11) when $N=0$.

A large set of algebraic solutions of Painlevé VI (and associated modular curves) have been obtained by many authors [38, 39, 40, 41, 42, 43. However, most of these results on algebraic solutions are for the canonical form the variable $y$ :

$$
\begin{gathered}
\frac{d^{2} y}{d t^{2}}=\frac{1}{2}\left(\frac{1}{y}+\frac{1}{1-y}+\frac{1}{y-t}\right)\left(\frac{d y}{d t}\right)^{2}-\left(\frac{1}{t}+\frac{1}{t-1}+\frac{1}{y-t}\right) \frac{d y}{d t} \\
+\frac{y(y-1)(y-t)}{t^{2}(t-1)^{2}}\left(\alpha+\beta \frac{t}{y^{2}}+\gamma \frac{t-1}{(y-1)^{2}}+\delta \frac{t(t-1)}{(y-t)^{2}}\right)
\end{gathered}
$$

There are several sets of $\alpha, \beta, \gamma, \delta$ which lead to the same equation 44 for $\sigma$. For

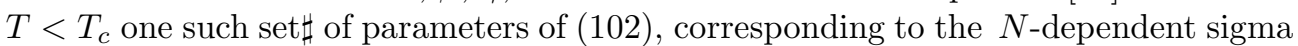
form (11), is :

$$
\alpha=\frac{1}{2}\left(N+\frac{1}{2}\right)^{2}, \quad \beta=-\frac{1}{2}\left(N-\frac{1}{2}\right)^{2}, \quad \gamma=\frac{1}{8}, \quad \delta=\frac{3}{8}
$$

It is interesting to make the connection between our results and those previously known algebraic solutions. Such a "dictionary" will be performed elsewher $\mathbb{\|}$, let us just give one simple example. The variable $y$ being a rational expression of $\sigma$ and its derivatives (see [4]), the algebraic solution (98) with (103), becomes $y=1-\sqrt{1-t}$ which is the well-known solution $8=\sqrt{t}$ (see [40]) under the change $t \rightarrow 1-t$, $y \rightarrow 1-y, \beta \rightarrow-\gamma$ and $\gamma \rightarrow-\beta$ which is a symmetry of (102).

\section{Conclusion}

The diagonal Ising two-point correlation functions can be expressed (see for instance [19, 45) as homogeneous polynomials of complete elliptic integral $E$ and $K$. These diagonal Ising correlations are $\lambda=1$ subcase of their $\lambda$-extensions $C(N, N ; \lambda)$ we considered in this paper. By (7) and (8) these polynomials of $E$ and $K$ are also expressed as infinite sums of the form factors $f_{M, N}^{(j)}$ 's which, themselves, are polynomials of $E$ and $K$. This yields a double infinity $(M, N)$ of remarkable identities on the complete elliptic integrals $E$ and $K$. Similarly, with the previous algebraic solutions for $\lambda=\cos (\pi m / n)$, one sees that an algebraic expression $C(N, N ; \cos (\pi m / n))$ (associated with a modular curve) can be written as an infinite sum of polynomials in $E$ and $K$. Each of these modular curves will provide a remarkable identity on the complete elliptic integrals $E$ and $K$.

Recalling relations like (5.7)-(5.11) of [14, all these identities can also be written in terms of the nome of the elliptic functions occurring in the Ising model. These identities, now, become remarkable identities on some infinite Gaussian sums, or on

$\|$ For $N=0$ this equation has been solved in terms of theta functions 40, 41, 42, has dihedral symmetry and has a countable number of algebraic solutions.

$\sharp$ To be considered when comparing with [40].

I We found that the $S L(2, Z)$ subgroup for $\tau^{n}$ for $n$ odd, (resp. $\tau^{2 n}$ for $n$ even) identify with the one for $y$ (see [37, 40.

$\S$ The solution $y=\sqrt{t}$ solves (102) for the parameters $(\alpha, \beta, \gamma, \delta)=(\alpha,-\alpha, 1 / 2-\delta, \delta)$. 
series expansions of theta functions, or, for large enough values of $N$, on Eisenstein series and other quasi-modular forms. We will describe, and analyze these identities in a forthcoming publication.

The calculations displayed in this paper can be seen as successful explicit examples of factorization of multiple integrals, providing examples of explicit calculations of the new mantra that "nested sums are Hopf algebras and thus multiple Feynmanlike integrals must factorize in terms of polynomial expressions of one-dimensional integrals". For our $j$-particle contributions of the diagonal correlation functions, the $C^{(j)}(M, N)$ 's, the fact that they are polynomial expressions of singled-out onedimensional integrals (the complete elliptic integrals $E$ and $K$ ) is understood in terms of direct sums of linear differential operators equivalent to symmetric powers of a singled-out linear differential operator. In the scaling limit, this direct sum structure, yielding polynomial expressions (that is the so-called "factorization of multiple integrals"), is lost: what remains is a Russian-doll structure of differential operators equivalent to symmetric powers of a singled-out differential operator.

The problem of the factorization of multiple integrals is, obviously, an important one for Feynman-like integrals. It also occurs on various calculations of correlation functions of integrable models, like the Heisenberg spin chain, where multiple integrals also occur. These factorizations are obtained by Boos and Korepin [46, 47] by adding to the integrand a successive set of anti-symmetric integrands (these anti-symmetric integrands being chosen in such a way that their multiple integral is zero). The combination of the initial integral with these new integrands yielding expressions depending on less variables, thus reducing the $n$-multiple integrals to a $(n-1)$-multiple integral. More recently, Boos et al. [48] also deduced factorization of multiple integrals representing the density matrix of the Heisenberg spin chain: the key ingredient, in the emergence of such factorization, is a functional identity on the integrand, this relation coming from the Bethe ansatz integrability of the model. The factorization of some multiple integrals can probably be seen as a consequence of some "YangBaxter integrability", it seems, however, to occur beyond this narrow framework. The Feynman-like integrals, where such factorization of some multiple integrals occurs, are not arbitrary holonomic expressions. What are the (more or less integrable) constraints one must impose on holonomic integrands such that their multiple integrals exhibit factorization, remains a fascinating open question [49]. A key point we have tried to promote here is that, instead of trying to calculate multiple integrals where the integrands have no free parameters, that is to say that the multiple integrals are just constants [50], we perform calculations on multiple integrals where the integrands do depend on one, or many, parameters. We can then use the holonomic structure.

In short: it is simpler to get multiple integrals that depend on one variable than obtaining their evaluation at a given value on this variable. This is typically a YangBaxter view point : it is easier to solve an integrable model with a spectral parameter that enables to describe the Yang-Baxter structure, than trying to solve that model for a given value of that parameter (quantum groups, knot theory, etc.). It is easier to solve the anisotropic Ising model than the isotropic one, and, similarly, it is easier to consider multiple integrals that depends on a variable, than evaluating constants [49] (polynomial expressions of $\zeta(3)$ ), $\zeta(5), \ldots$ ) corresponding to these multiple integrals at a given value of that parameter: this way of looking at the problem enables to see the emergence of highly non trivial algebraic structures on linear differential operators, that are a very efficient and powerful tool of experimental mathematics, and other formal calculations, to study factorizations of multiple integrals. 
Acknowledgments: We thank Prof. R. J. Baxter for interesting comments on this work, and Prof. C.A. Tracy for generously providing some of his notes on calculations he performed on integrals related to the Ising model. We thank A. Its for pointing out some important references on Picard solutions to PVI and algebraic solutions derived from them. We do thank J-A. Weil for illuminating comments on the scaling limits of differential operators, M. Rybowicz for help in some of our extensive formal calculations, V.E. Korepin for sharing his knowledge of multiple integrable calculations, and N. Witte for interesting information on the Garnier systems. One of us (BM) has been partially supported by NSF grant DMR-0302758. One of us (BM) thanks J-M. Maillet for hospitality at Ecole Normale Supérieure in Lyon where part of this work was done. One of us (NZ) would like to acknowledge kind hospitality at the LPTMC where part of this work has been completed. One of us (JMM) thanks Stony Brook and the MASCOS (Melbourne) where part of this work was performed.

\section{Appendix A. Differential operators $L_{j}(N)$}

The linear differential operators $L_{j}(N)$ have the following form:

$$
L_{j}(N)=\sum_{n=0}^{n_{0}} \frac{N^{2 n}}{t^{2 n}} \cdot\left(\sum_{k=0}^{j-2 k} \frac{P_{n, k}^{(j)}(t)}{(t(t-1))^{k}} D t^{j-2 n-k}\right)
$$

where $n_{0}=(j-1) / 2$ for $j$ odd, and $n_{0}=j / 2$ for $j$ even. The polynomials $P_{n, k}^{(j)}(t)$ are of degree $k$ in $t$.

Appendix A.1. $P_{n, k}^{(5)}(t)$

$$
\begin{aligned}
& P_{0,0}^{(5)}=1, \quad P_{0,1}^{(5)}=40 t-20, \quad P_{0,2}^{(5)}=-563 t+558 t^{2}+118, \\
& P_{0,3}^{(5)}=\frac{4291}{2} t-\frac{10169}{2} t^{2}+3320 t^{3}-220, \\
& P_{0,4}^{(5)}=80+10848 t^{2}-16978 t^{3}+8180 t^{4}-2227 t, \\
& P_{0,5}^{(5)}=4\left(85-1139 t+3672 t^{2}-4250 t^{3}+1600 t^{4}\right) t, \\
& P_{1,0}^{(5)}=-5, \quad P_{1,1}^{(5)}=-91 t+59, \quad P_{1,2}^{(5)}=-469 t^{2}+626 t-181, \\
& P_{1,3}^{(5)}=144-840 t+1368 t^{2}-656 t^{3}, \quad P_{2,0}^{(5)}=4, \quad P_{2,1}^{(5)}=16 t-16
\end{aligned}
$$

Appendix A.2. $P_{n, k}^{(6)}(t)$

$$
\begin{aligned}
& P_{0,0}^{(6)}=1, \quad P_{0,1}^{(6)}=70 t-35, \quad P_{0,2}^{(6)}=\frac{7427}{4} t^{2}-\frac{7427}{4} t+413, \\
& P_{0,3}^{(6)}=2(2 t-1)\left(5912 t^{2}-5912 t+979\right), \\
& P_{0,4}^{(6)}=\frac{2410523}{16} t^{4}-\frac{2410523}{8} t^{3}+\frac{3200163}{16} t^{2}-\frac{98705}{2} t+3383, \\
& P_{0,5}^{(6)}=\frac{1}{16}(2 t-1)\left(3585925 t^{4}-7171850 t^{3}+4326453 t^{2}-740528 t+19600\right), \\
& P_{0,6}^{(6)}=\frac{625}{64} t(t-1)\left(48841 t^{4}-97682 t^{3}+63549 t^{2}-14708 t+784\right),
\end{aligned}
$$


Holonomy of the Ising model form factors

$$
\begin{aligned}
& P_{1,0}^{(6)}=-\frac{35}{4}, \quad P_{1,1}^{(6)}=-336 t+\frac{413}{2}, \\
& P_{1,2}^{(6)}=-\frac{34799}{8} t^{2}+\frac{43231}{8} t-\frac{6133}{4}, \\
& P_{1,3}^{(6)}=-\frac{88609}{4} t^{3}+41823 t^{2}-\frac{96849}{4} t+\frac{16691}{4}, \\
& P_{1,4}^{(6)}=-\frac{25}{64}(t-1)\left(94091 t^{3}-146523 t^{2}+67548 t-9216\right), \\
& P_{2,0}^{(6)}=\frac{259}{16}, \quad P_{2,1}^{(6)}=\frac{1917}{8} t-\frac{3159}{16}, \\
& P_{2,2}^{(6)}=\frac{125}{64}(407 t-272)(t-1), \quad P_{3,0}^{(6)}=-\frac{225}{64}
\end{aligned}
$$

Appendix A.3. $P_{n, k}^{(7)}(t)$

$$
\begin{aligned}
P_{0,0}^{(7)} & =1, \quad P_{0,1}^{(7)}=-56+112 t, \quad P_{0,2}^{(7)}=5012 t^{2}-5026 t+1148, \\
P_{0,3}^{(7)} & =-10736+79727 t-174373 t^{2}+115544 t^{3}, \\
P_{0,4}^{(7)} & =46172-548736 t+2042953 t^{2}-2975244 t^{3}+1472828 t^{4}, \\
P_{0,5}^{(7)} & =-78640+1605642 t-9634279 t^{2}+23975501 t^{3} \\
\quad- & 26144958 t^{4}+10305440 t^{5}, \\
P_{0,6}^{(7)} & =29160-1616078 t+\frac{67624527}{4} t^{2} \\
- & \frac{136608085}{2} t^{3}+\frac{511207495}{4} t^{4}-111249042 t^{5}+36334360 t^{6}, \\
P_{0,7}^{(7)} & =\frac{9}{2} t\left(59940-1665037 t+11865715 t^{2}-36308026 t^{3}+54466294 t^{4}\right. \\
- & \left.39393900 t^{5}+10951200 t^{6}\right), \\
P_{1,0}^{(7)} & =-14, \quad P_{1,1}^{(7)}=-966 t+574, \\
P_{1,2}^{(7)} & =-24712 t^{2}+29686 t-8248, \\
P_{1,3}^{(7)} & =-290812 t^{3}+530547 t^{2}-299013 t+51188, \\
P_{1,4}^{(7)} & =-1561136 t^{4}+3851903 t^{3}-3309480 t^{2}+1156221 t-136440, \\
P_{1,5}^{(7)} & =129600-\frac{22166415}{2} t^{3}+\frac{18989235}{2} t^{4}+\frac{11893977}{2} t^{2} \\
& -\frac{2902725}{2} t-3028104 t^{5}, \\
P_{2,0}^{(7)} & =49, \quad P_{2,1}^{(7)}=1686 t-1254, \quad P_{2,2}^{(7)}=17887 t^{2}-27026 t+9679, \\
P_{2,3}^{(7)} & =-22761-133569 t^{2}+57753 t^{3}+98253 t, \\
P_{3,0}^{(7)} & =-36, \quad P_{3,1}^{(7)}=324-324 t
\end{aligned}
$$

Appendix A.4. $P_{n, k}^{(8)}(t)$

$$
P_{0,0}^{(8)}=1, \quad P_{0,1}^{(8)}=168 t-84, \quad P_{0,2}^{(8)}=11697 t^{2}-11697 t+2730,
$$




$$
\begin{aligned}
& P_{0,3}^{(8)}=2(2 t-1)\left(109862 t^{2}-109862 t+21881\right), \\
& P_{0,4}^{(8)}=\frac{77675835}{8} t^{4}-\frac{77675835}{4} t^{3}+\frac{108450015}{8} t^{2}-\frac{7693545}{2} t+364365 \text {, } \\
& P_{0,5}^{(8)}=\frac{1}{4}(2 t-1)\left(257365313 t^{4}-514730626 t^{3}+340542345 t^{2}\right. \\
& -83177032 t+6033464) \\
& P_{0,6}^{(8)}=2610671-\frac{135579123}{2} t+\frac{4351723053}{8} t^{2}-\frac{31150612733}{16} t^{3} \\
& -\frac{47738959467}{16} t^{5}+\frac{55357772589}{16} t^{4}+\frac{15912986489}{16} t^{6}, \\
& P_{0,7}^{(8)}=\frac{1}{8}(2 t-1)\left(16309728941 t^{6}-48929186823 t^{5}+54824769942 t^{4}\right. \\
& \left.-28100895179 t^{3}+6440184015 t^{2}-544600896 t+8016008\right), \\
& P_{0,8}^{(8)}=\frac{2401}{256} t(t-1)\left(719580625 t^{6}-2158741875 t^{5}+2496751275 t^{4}\right. \\
& \left.-1395599425 t^{3}+383051976 t^{2}-45042576 t+1308736\right) \text {, } \\
& P_{1,0}^{(8)}=-21, \quad P_{1,1}^{(8)}=-2352 t+1365, \\
& P_{1,2}^{(8)}=-\frac{414555}{4} t^{2}+\frac{483843}{4} t-33315 \text {, } \\
& P_{1,3}^{(8)}=-2290461 t^{3}+4034358 t^{2}-2237787 t+386664 \text {, } \\
& P_{1,4}^{(8)}=-\frac{426526863}{16} t^{4}+\frac{504203159}{8} t^{3}-\frac{845513895}{16} t^{2}+\frac{36865265}{2} t \\
& \text { - 2230431, } \\
& P_{1,5}^{(8)}=-\frac{616586181}{4} t^{5}+\frac{7342474719}{16} t^{4}-\frac{4139827129}{8} t^{3} \\
& +\frac{4378085671}{16} t^{2}-67155042 t+6072033 \\
& P_{1,6}^{(8)}=-\frac{49}{64}(t-1)\left(449304249 t^{5}-1168884874 t^{4}+1134316077 t^{3}\right. \\
& \left.-509448428 t^{2}+105774112 t-8294400\right) \text {, } \\
& P_{2,0}^{(8)}=\frac{987}{8}, \quad P_{2,1}^{(8)}=\frac{15993}{2} t-\frac{22299}{4}, \\
& P_{2,2}^{(8)}=\frac{2933043}{16} t^{2}-\frac{4128099}{16} t+\frac{696405}{8} \text {, } \\
& P_{2,3}^{(8)}=\frac{7002915}{4} t^{3}-\frac{14949545}{4} t^{2}+\frac{10236397}{4} t-\frac{4465707}{8}, \\
& P_{2,4}^{(8)}=\frac{343}{128}(t-1)\left(2179797 t^{3}-4103797 t^{2}+2457908 t-468864\right) \text {, } \\
& P_{3,0}^{(8)}=-\frac{3229}{16}, \quad P_{3,1}^{(8)}=-\frac{21963}{4} t+\frac{76827}{16}, \\
& P_{3,2}^{(8)}=-\frac{343}{64}(6607 t-5032)(t-1), \quad P_{4,0}^{(8)}=\frac{11025}{256}
\end{aligned}
$$


Appendix A.5. $P_{n, k}^{(9)}(t)$

$$
\begin{aligned}
& P_{0,0}^{(9)}=1, \quad P_{0,1}^{(9)}=-120+240 t, \quad P_{0,2}^{(9)}=5796-24546 t+24516 t^{2}, \\
& P_{0,3}^{(9)}=-145528+991701 t-2099751 t^{2}+1396208 t^{3}, \\
& P_{0,4}^{(9)}=2045004-20325858 t+69369177 t^{2}-97902648 t^{3}+48749364 t^{4} \text {, } \\
& P_{0,5}^{(9)}=-16074560+225525578 t-1125696965 t^{2}+2565535675 t^{3} \\
& -2714936962 t^{4}+1079617840 t^{5} \\
& P_{0,6}^{(9)}=66126712-1333788966 t+\frac{37765468163}{4} t^{2}-\frac{63124281313}{2} t^{3} \\
& +\frac{216166206483}{4} t^{4}-45734526046 t^{5}+15125870712 t^{6}, \\
& P_{0,7}^{(9)}=-118102672+3823928460 t-\frac{78510959875}{2} t^{2}+\frac{374049548401}{2} t^{3} \\
& -471178501099 t^{4}+646530989251 t^{5}-455734056216 t^{6} \\
& +128906004992 t^{7}, P_{0,8}^{(9)}=47071232-4139526516 t+\frac{138902716891}{2} t^{2} \\
& -484196478836 t^{3}+\frac{3495148889889}{2} t^{4}-3539969007392 t^{5} \\
& +4054878125399 t^{6}-2448333931344 t^{7}+604418968592 t^{8} \text {, } \\
& P_{0,9}^{(9)}=80 t\left(9561344-427020633 t+4937178194 t^{2}-26308505171 t^{3}\right. \\
& +76760779797 t^{4}-130255661861 t^{5}+128108854250 t^{6} \\
& \left.-67626000000 t^{7}+14796800000 t^{8}\right), \quad P_{1,0}^{(9)}=-30, \\
& P_{1,1}^{(9)}=-5082 t+2898, P_{1,2}^{(9)}=-352662 t^{2}+404466 t-110238, \\
& P_{1,3}^{(9)}=-12963996 t^{3}+22438245 t^{2}-12306435 t+2123604, \\
& P_{1,4}^{(9)}=-271930980 t^{4}+631696597 t^{3}-523169724 t^{2}+181823257 t \\
& -22193940, P_{1,5}^{(9)}=-3245449704 t^{5}+\frac{18983501249}{2} t^{4} \\
& -\frac{21116262613}{2} t^{3}+\frac{11093266991}{2} t^{2}-\frac{2731643299}{2} t+125146416, \\
& P_{1,6}^{(9)}=-20342103432 t^{6}+71951600804 t^{5}-100847772344 t^{4} \\
& +71245445309 t^{3}-26573340926 t^{2}+4930067225 t-354631488 \\
& P_{1,7}^{(9)}=406425600-363525018400 t^{5}-6762200560 t+46013156464 t^{2} \\
& +214239244800 t^{6}-51475353600 t^{7}+324098542224 t^{4} \\
& -162977694704 t^{3}, \quad P_{2,0}^{(9)}=273, \quad P_{2,1}^{(9)}=29490 t-19650, \\
& P_{2,2}^{(9)}=1217265 t^{2}-1636902 t+528465 \text {, } \\
& P_{2,3}^{(9)}=23917695 t^{3}-48731759 t^{2}+31834675 t-6637935 \text {, } \\
& P_{2,4}^{(9)}=222934641 t^{4}-612371540 t^{3}+607781638 t^{2}-257369288 t \\
& +39119361, P_{2,5}^{(9)}=734599360 t-2367055040 t^{2}-2730725376 t^{4}
\end{aligned}
$$




$$
\begin{aligned}
& -87745536+785703936 t^{5}+3664705792 t^{3}, \\
& P_{3,0}^{(9)}=-820, P_{3,1}^{(9)}=-46428 t+37212, \\
& P_{3,2}^{(9)}=-839284 t^{2}+1358312 t-535156, \\
& P_{3,3}^{(9)}=2455552-9463296 t+11831808 t^{2}-4814848 t^{3}, \\
& P_{4,0}^{(9)}=576, \quad P_{4,1}^{(9)}=-9216+9216 t
\end{aligned}
$$

Appendix A.6. $P_{n, k}^{(10)}(t)$

$$
\begin{aligned}
& P_{0,0}^{(10)}=1, P_{0,1}^{(10)}=-165+330 t, P_{0,2}^{(10)}=11286-\frac{189189}{4} t+\frac{189189}{4} t^{2}, \\
& P_{0,3}^{(10)}=440(-1+2 t)\left(4400 t^{2}-4400 t+947\right) \text {, } \\
& P_{0,4}^{(10)}=9053979-85922628 t+\frac{2289151821}{8} t^{2}-\frac{1601770797}{4} t^{3} \\
& +\frac{1601770797}{8} t^{4} \\
& P_{0,5}^{(10)}=\frac{1}{8}(2 t-1)\left(27291921049 t^{4}-54583842098 t^{3}\right. \\
& \left.+37651935321 t^{2}-10360014272 t+946138408\right) \text {, } \\
& P_{0,6}^{(10)}=907059937-\frac{63513668189}{4} t+\frac{414126483423}{4} t^{2} \\
& -\frac{10567258749853}{32} t^{3}+\frac{17677263640199}{32} t^{4}-\frac{14872361118327}{32} t^{5} \\
& +\frac{4957453706109}{32} t^{6} \\
& P_{0,7}^{(10)}=\frac{1}{8}(2 t-1)\left(9240801571631 t^{6}\right. \\
& -27722404714893 t^{5}+32221157315067 t^{4}-18238306771979 t^{3} \\
& \left.+5175369000414 t^{2}-676616400240 t+30201789392\right) \text {, } \\
& P_{0,8}^{(10)}=7010881775-\frac{537428072635}{2} t+\frac{53588823341945}{16} t^{2} \\
& -\frac{320491542697265}{16} t^{3}+\frac{16973016403001045}{256} t^{4} \\
& -\frac{8195527196507945}{64} t^{5}+\frac{18373024724608855}{128} t^{6} \\
& -\frac{5532574254401525}{64} t^{7}+\frac{5532574254401525}{256} t^{8}, \\
& P_{0,9}^{(10)}=\frac{5}{256}(2 t-1)\left(2925753951778285 t^{8}-11703015807113140 t^{7}\right. \\
& +19103849088522126 t^{6}-16350991940670388 t^{5} \\
& +7838575034697949 t^{4}-2079015276577248 t^{3}+280113055050736 t^{2} \\
& -15268105688320 t+148553637120) \text {, } \\
& P_{0,10}^{(10)}=\frac{32805}{1024} t(t-1)\left(8079810760125 t^{8}-32319243040500 t^{7}\right.
\end{aligned}
$$




$$
\begin{aligned}
& +53723369995078 t^{6}-48052759343484 t^{5}+24975072368117 t^{4} \\
& -7567996044344 t^{3}+1257903576048 t^{2}-96158271040 t \\
& +1833995520), \quad P_{1,0}^{(10)}=-\frac{165}{4}, \quad P_{1,1}^{(10)}=-10032 t+5643, \\
& P_{1,2}^{(10)}=-\frac{4116057}{4} t^{2}+\frac{4646169}{4} t-\frac{629409}{2}, \\
& P_{1,3}^{(10)}=-\frac{116107101}{2} t^{3}+98646966 t^{2}-\frac{107291349}{2} t+\frac{18577449}{2}, \\
& P_{1,4}^{(10)}=-\frac{62944154655}{32} t^{4}+\frac{71575322887}{16} t^{3}-\frac{117218126643}{32} t^{2} \\
& +\frac{10187763521}{8} t-\frac{630658425}{4} \\
& P_{1,5}^{(10)}=-\frac{163658617341}{4} t^{5}+\frac{1868493139019}{16} t^{4}-\frac{1024185567025}{8} t^{3} \\
& +\frac{1072700805259}{16} t^{2}-16676053189 t+\frac{3132363327}{2}, \\
& P_{1,6}^{(10)}=-\frac{32591037777225}{64} t^{6}+\frac{112104944104795}{64} t^{5}-\frac{154297814907493}{64} t^{4} \\
& +\frac{108234764940653}{64} t^{3}-\frac{20292699152369}{32} t^{2} \\
& +\frac{958545911705}{8} t-\frac{35460987675}{4} \\
& P_{1,7}^{(10)}=-\frac{110727690476325}{32} t^{7}+\frac{446374095368415}{32} t^{6} \\
& -\frac{740746002842197}{32} t^{5}+\frac{163197508324913}{8} t^{4}-\frac{82052435490193}{8} t^{3} \\
& +\frac{93651745559635}{32} t^{2}-\frac{1748299590545}{4} t+\frac{105491089125}{4}, \\
& P_{1,8}^{(10)}=-\frac{6561}{1024}(t-1)\left(1530421397125 t^{7}-5554611547375 t^{6}\right. \\
& +8232623167111 t^{5}-6428537243541 t^{4}+2844044623496 t^{3} \\
& \left.-711552088080 t^{2}+93740238400 t-5138022400\right) \text {, } \\
& P_{2,0}^{(10)}=\frac{4389}{8}, \quad P_{2,1}^{(10)}=\frac{364353}{4} t-\frac{469491}{8}, \\
& P_{2,2}^{(10)}=\frac{194340135}{32} t^{2}-\frac{251951799}{32} t+\frac{19747365}{8}, \\
& P_{2,3}^{(10)}=\frac{830796045}{4} t^{3}-406585301 t^{2}+\frac{1027545163}{4} t-\frac{208821765}{4}, \\
& P_{2,4}^{(10)}=\frac{490847729943}{128} t^{4}-\frac{645152215543}{64} t^{3}+\frac{1232916142207}{128} t^{2} \\
& -\frac{63246182437}{16} t+\frac{4689902523}{8} \\
& P_{2,5}^{(10)}=\frac{2314309478331}{64} t^{5}-\frac{15325677165945}{128} t^{4}+\frac{1231151157175}{8} t^{3} \\
& -\frac{12252529589789}{128} t^{2}+\frac{230003188957}{8} t-\frac{26662508757}{8}, \\
& P_{2,6}^{(10)}=\frac{729}{512}(t-1)\left(95338644413 t^{5}-286574346250 t^{4}\right.
\end{aligned}
$$


Holonomy of the Ising model form factors

$$
\begin{aligned}
& \left.+332882516705 t^{3}-186665173556 t^{2}+50609686768 t-5337817088\right), \\
& P_{3,0}^{(10)}=-\frac{86405}{32}, \quad P_{3,1}^{(10)}=-\frac{1071807}{4} t+\frac{3230007}{16}, \\
& P_{3,2}^{(10)}=-\frac{616031665}{64} t^{2}+\frac{934953233}{64} t-\frac{173220155}{32}, \\
& P_{3,3}^{(10)}=-\frac{4731457901}{32} t^{3}+\frac{2715664857}{8} t^{2}-\frac{8133623529}{32} t+\frac{1981312349}{32}, \\
& P_{3,4}^{(10)}=-\frac{2187}{512}(t-1)\left(191281007 t^{3}-399820191 t^{2}+271766508 t\right. \\
& -60099968), \\
& P_{4,0}^{(10)}=\frac{1057221}{256}, \quad P_{4,1}^{(10)}=\frac{23053617}{128} t-\frac{41642109}{256}, \\
& P_{4,2}^{(10)}=\frac{2187}{1024}(892447 t-727072)(t-1), \quad P_{5,0}^{(10)}=-\frac{893025}{1024}
\end{aligned}
$$

\section{Appendix B. Direct sum structure}

We display the fourth order differential operator $M_{4}(N)$ introduced in sec. 4.3 for successive values of $N$ :

$$
\begin{aligned}
& M_{4}(0)=D t^{4}+2 \frac{(2 t-1)\left(2 t^{2}-2 t+3\right)}{\left(t^{2}-t+1\right)(t-1) t} \cdot D t^{3} \\
& +\frac{1}{2} \frac{\left(-73 t+14+102 t^{2}-58 t^{3}+29 t^{4}\right)}{\left(t^{2}-t+1\right)(t-1)^{2} t^{2}} \cdot D t^{2} \\
& +\frac{1}{2} \frac{(2 t-1)\left(5 t^{4}-10 t^{3}+27 t^{2}-22 t+2\right)}{\left(t^{2}-t+1\right)(t-1)^{3} t^{3}} \cdot D t \\
& +\frac{1}{16} \frac{t^{4}-2 t^{3}+42 t^{2}-41 t+4}{\left(t^{2}-t+1\right)(t-1)^{3} t^{3}} \\
& M_{4}(1)=D t^{4}+2 \frac{P_{3}}{(t-1) t \cdot P_{4}} \cdot D t^{3}+\frac{1}{2} \frac{P_{2}}{(t-1)^{2} t^{2} P_{4}} \cdot D t^{2} \\
& \quad+\frac{P_{1}}{(t-1)^{3} t^{3} \cdot P_{4}} \cdot D t+\frac{1}{16} \frac{P_{0}}{(t-1)^{3} t^{4} \cdot P_{4}}
\end{aligned}
$$

where

$$
\begin{aligned}
P_{0} & =256 t^{6}-560 t^{5}+312 t^{4}-143 t^{3}+227 t^{2}-72 t-16, \\
P_{1} & =64 t^{7}-856 t^{6}+2826 t^{5}-4087 t^{4}+2978 t^{3}-1098 t^{2}+182 t-8, \\
P_{2} & =208+7807 t^{2}-14253 t^{3}+12412 t^{4}-4624 t^{5}+448 t^{6}, \\
P_{3} & =64 t^{5}-556 t^{4}+1225 t^{3}-1078 t^{2}+396 t-48, \\
P_{4} & =16+209 t^{2}-120 t^{3}+16 t^{4}-120 t, \\
M_{4}(2) & =D t^{4}+2 \frac{P_{3}}{t(t-1) P_{4}} \cdot D t^{3}+\frac{1}{2} \frac{P_{2}}{t^{2}(t-1)^{2} P_{4}} \cdot D t^{2} \\
& +\frac{1}{2} \frac{P_{1}}{t^{3}(t-1)^{3} P_{4}} \cdot D t+\frac{1}{16} \frac{P_{0}}{t^{4}(t-1)^{3} P_{4}}
\end{aligned}
$$


where the corresponding $P_{i}$ 's read :

$$
\begin{aligned}
& P_{0}=-1344 t^{11}+10752 t^{10}+139321 t^{9}-721147 t^{8}+1888781 t^{7} \\
& \quad-3452437 t^{6}+4219535 t^{5}-3184189 t^{4}+1330028 t^{3} \\
& \quad-202384 t^{2}-34048 t+7168 \\
& P_{1}=448 t^{11}+4256 t^{10}+56658 t^{9}-519911 t^{8}+1502563 t^{7}-2077796 t^{6} \\
& \quad+1426525 t^{5}-372047 t^{4}-39536 t^{3}+5418 t^{2}+14336 t-896, \\
& P_{2}=4928 t^{10}-37632 t-1394407 t^{3}+4810853 t^{4}-8001289 t^{5} \\
& \quad+6880493 t^{6}+415793 t^{8}-2881207 t^{7}+16128 t^{9}+11648 \\
& \quad+174818 t^{2}, \\
& P_{3}=1344 t^{9}+1568 t^{8}+65828 t^{7}-382102 t^{6}+760238 t^{5}-702181 t^{4} \\
& \quad+302183 t^{3}-46627 t^{2}+1568 t-1792, \\
& P_{4}=448 t^{8}+448 t^{7}+16513 t^{6}-81242 t^{5}+127675 t^{4}-81242 t^{3} \\
& \quad+16513 t^{2}+448 t+448
\end{aligned}
$$

\section{Appendix C. The form factors $f_{N, N}^{(j)}$}

In order to check all the results displayed in this paper, we have performed a large number of series expansions. Even the series expansions obtained recursively, order by order, from the sigma form of Painlevé VI (11) in sec. 3, were checked against series expansions obtained independently. Some were based on extremely large series expansions, not in $s$ or $t$, but in the nome of elliptic functions (see (5.7)-(5.11) of [14]), others were obtained from series expansions with hypergeometric functions coefficients.

Actually our new simple integral representations (35), (36) are of a great help to produce large series expansions for the quantities $f_{N, N}^{(2 n)}(t)$ and $f_{N, N}^{(2 n+1)}(t)$. This amounts to expanding only the $\left(1-t x_{2 j-1} x_{2 k}\right)^{-2}$ term in (36). Recalling the Euler representation of the hypergeometric functions [30]:

$$
\begin{aligned}
& F(a, b, c ; t)=\frac{\Gamma(c)}{\Gamma(c-b) \Gamma(b)} \\
& \int_{0}^{1} x^{b-1}(1-x)^{c-b-1}(1-x t)^{-a} d x
\end{aligned}
$$

one can rewrite, alternatively, these integral representations (35), (36) expansions of $f_{N, N}^{(2 n)}(t)$ and $f_{N, N}^{(2 n+1)}(t)$, as nested sums of products of hypergeometric functions. By expanding the factor $\left(1-t x_{1} x_{2}\right)^{-2}$ in a power series in $t$ we obtain

$$
\begin{aligned}
& f_{N, N}^{(2)}(t)=t^{N+1} \cdot \sum_{j=0}^{\infty}(j+1) t^{j} \cdot \frac{(1 / 2)_{N+j}(3 / 2)_{N+j}}{4(N+j+1) !^{2}} \times \\
& F(-1 / 2, N+j+1 / 2, N+j+2 ; t) F(1 / 2, N+j+3 / 2, N+j+2 ; t)
\end{aligned}
$$

The series expansions for $h_{2 j}(N, N)(t)$ 's and the $h_{2 j+1}(N, N)(t)$ 's agree with the series expansions for the $\hat{C}^{j}(N, N)$ 's and with the series expansions for the $f_{N, N}^{(2 j)}$ 's and $f_{N, N}^{(2 j+1)}$ 's. In this Appendix, we display the $f_{N, N}^{(2 j)}$ 's and $f_{N, N}^{(2 j+1)}$ 's for some $j$ and some $N$. 
Appendix C.1. $f_{N, N}^{(1)}$ and $f_{N, N}^{(2)}$

The $f_{N, N}^{(1)}$ 's are given in the text by (41) and (44). The $f_{N, N}^{(2)}$ 's are given explicitly as a function of $K$ and $E$ in the text.

Appendix C.2. $f_{N, N}^{(3)}$

The $f_{N, N}^{(3)}$ 's read for $N=0, \cdots, 4$ :

$$
\begin{aligned}
& 6 f_{0,0}^{(3)}=K-(t-2) K^{3}-3 K^{2} E \\
& 6 t^{1 / 2} f_{1,1}^{3}=4(K-E)-6 K^{2} E-(2 t-3) K^{3}+3 K E^{2} \\
& 18 t f_{2,2}^{(3)}=7(t+2) K-14(t+1) E+24 E^{3} \\
& \quad+3\left(2 t^{2}-11 t+2\right) E K^{2}-3\left(t^{2}-2\right) K^{3}+36(t-1) K E^{2} \\
& 270 t^{5 / 2} f_{3,3}^{(3)}=-30\left(8 t^{2}+7 t+8\right) t \cdot E+30\left(4 t^{2}+3 t+8\right) K \\
& \quad-\left(72 t^{4}-158 t^{3}+189 t^{2}-156 t+8\right) K^{3} \\
& \quad+6\left(24 t^{4}-108 t^{3}+29 t^{2}-6 t+4\right) E K^{2} \\
& \quad+3\left(232 t^{3}-111 t^{2}-180 t-8\right) E^{2} K \\
& \quad+4(t+1)\left(2 t^{2}+103 t+2\right) t E^{3} \\
& 47250 t^{4} f_{4,4}^{(3)}=975(3 t+4)\left(8 t^{2}-5 t+12\right) t^{2} K \\
& \quad-7800 t^{2}(t+1)\left(6 t^{2}-t+6\right) E \\
& \quad-\left(16216 t^{6}-32109 t^{5}+4218 t^{4}+38472 t^{3}-38064 t^{2}+3264 t+128\right) K^{3} \\
& \quad+3\left(10832 t^{6}-43424 t^{5}+4925 t^{4}+13248 t^{3}-10112 t^{2}+3328 t+128\right) E K^{2} \\
& \quad-48\left(4 t^{6}-2885 t^{5}+939 t^{4}+1510 t^{3}+1792 t^{2}+212 t+8\right) E^{2} K \\
& \quad+16\left(8 t^{6}+216 t^{5}+4893 t^{4}+5464 t^{3}+4893 t^{2}+216 t+8\right) E^{3}
\end{aligned}
$$

Appendix C.3. $f_{N, N}^{(4)}$

Some of the $f_{N, N}^{(4)}$ 's read:

$$
\begin{aligned}
& 24 f_{0,0}^{(4)}=4(K-E) \cdot K-(2 t-3) K^{4}-6 K^{3} E+3 K^{2} E^{2} \\
& 24 f_{1,1}^{(4)}=9-30 K E-10(t-2) K^{2} \\
& \quad+\left(t^{2}-6 t+6\right) K^{4}+15 K^{2} E^{2}+10(t-2) K^{3} E \\
& 72 t \cdot f_{2,2}^{(4)}=72 t-32(1+t) E^{2} \\
& \quad-16\left(2+6 t^{2}-11 t\right) K^{2}-16(15 t-4) K E \\
& \quad+\left(24 t^{3}-98 t^{2}+113 t-36\right) K^{4} \\
& \quad+12(9+t) E^{3} K+3(71 t-60) K^{2} E^{2} \\
& \quad+2\left(66+74 t^{2}-157 t\right) E K^{3}-24 E^{4} \\
& 1080 t^{2} f_{3,3}^{(4)}=22\left(8 t^{3}-319 t^{2}+112 t+16\right) K E \\
& \quad-88(1+t)\left(2 t^{2}+13 t+2\right) E^{2}
\end{aligned}
$$


Holonomy of the Ising model form factors

$$
\begin{aligned}
& +\left(957 t^{4}-3646 t^{3}+4230 t^{2}-1488 t-8\right) K^{4} \\
& +8\left(46 t^{3}+51 t^{2}+543 t-110\right) E^{3} K \\
& +3\left(16 t^{4}-72 t^{3}+2537 t^{2}-2704 t+272\right) K^{2} E^{2} \\
& -6\left(8 t^{4}-903 t^{3}+1934 t^{2}-988 t+40\right) E K^{3} \\
& +24\left(13+13 t^{2}-28 t\right) E^{4} \\
& -22\left(137 t^{3}-242 t^{2}+52 t+8\right) K^{2}+2025 t^{2}
\end{aligned}
$$

Appendix C.4. $f_{N, N}^{(5)}$

We give some $f_{N, N}^{(5)}$ 's:

$$
\begin{array}{rl}
120 & t^{1 / 2} f_{1,1}^{(5)}=64 \cdot(K-E) \\
& -20(2 t-3) K^{3}-120 E K^{2}+60 E^{2} K \\
& +(4 t-5)(2 t-3) K^{5}+15(2 t-3) K^{4} E+45 K^{3} E^{2}-15 K^{2} E^{3} \\
360 & t f_{2,2}^{(5)}=149(t+2) K-298(t+1) E \\
& +720 E^{3}-90\left(t^{2}-2\right) K^{3} \\
& +90\left(2 t^{2}-11 t+2\right) E K^{2}+1080(t-1) E^{2} K \\
& +\left(5 t^{3}+28 t^{2}-90 t+60\right) K^{5}-10\left(t^{3}-16 t^{2}+24 t-4\right) E K^{4} \\
& -5\left(32 t^{2}-179 t+122\right) K^{3} E^{2}-30(19 t-29) K^{2} E^{3}-360 E^{4} K \\
5400 & t^{5 / 2} f_{3,3}^{(5)}=792\left(4 t^{2}+3 t+8\right) t \cdot K-792 t\left(8 t^{2}+7 t+8\right) \cdot E \\
& -40\left(72 t^{4}-158 t^{3}+189 t^{2}-156 t+8\right) K^{3} \\
& +240\left(24 t^{4}-108 t^{3}+29 t^{2}-6 t+4\right) E K^{2} \\
& +120\left(232 t^{3}-111 t^{2}-180 t-8\right) E^{2} K \\
& +160(t+1)\left(2 t^{2}+103 t+2\right) E^{3} \\
& +5\left(96 t^{5}-520 t^{4}+1310 t^{3}-1589 t^{2}+800 t-88\right) K^{5} \\
& +5\left(424-2488 t+5051 t^{2}-4962 t^{3}+2008 t^{4}-192 t^{5}\right) E K^{4} \\
& -5\left(1984 t^{4}-9228 t^{3}+9423 t^{2}-3272 t+816\right) E^{2} K^{3} \\
& +5\left(784-4104 t+11697 t^{2}-6056 t^{3}\right) E^{3} K^{2} \\
& -40\left(2 t^{3}+738 t^{2}-567 t+47\right) E^{4} K \\
+ & 360\left(t^{2}-28 t+1\right) E^{5}
\end{array}
$$

Appendix C.5. $\quad f_{N, N}^{(6)}$

Some $f_{N, N}^{(6)}$ read:

$$
\begin{aligned}
720 & f_{1,1}^{(6)}=225-259(t-2) K^{2}-777 K E \\
& -105 K^{3} E^{3}+525 K^{2} E^{2}+350(t-2) E K^{3}+35\left(6+t^{2}-6 t\right) K^{4} \\
& -21\left(6+t^{2}-6 t\right) E K^{5}-105(t-2) E^{2} K^{4} \\
& -(t-2)\left(t^{2}-10 t+10\right) K^{6}
\end{aligned}
$$


Holonomy of the Ising model form factors

$$
\begin{aligned}
& 2160 t f_{2,2}^{(6)}=2160 t-544(15 t-4) K E \\
& \quad-1088(1+t) \cdot E^{2}-544\left(6 t^{2}-11 t+2\right) \cdot K^{2} \\
& \quad+50\left(24 t^{3}-98 t^{2}+113 t-36\right) K^{4}-1200 E^{4}+600(9+t) E^{3} K \\
& \quad+150(71 t-60) K^{2} E^{2}+100\left(66+74 t^{2}-157 t\right) E K^{3} \\
& \quad+360 K E^{5}-15(235 t-264) K^{3} E^{3} \\
& \quad+3\left(720-1889 t+1490 t^{2}-344 t^{3}\right) \cdot E K^{5} \\
& \quad-90(21+t) E^{4} K^{2}-45\left(92+74 t^{2}-173 t\right) \cdot E^{2} K^{4} \\
& \quad-3\left(32 t^{4}-220 t^{3}+504 t^{2}-467 t+150\right) K^{6}
\end{aligned}
$$

All these $f_{N, N}^{(j)}$ displayed when expanded have their leading coefficients starting as given in (35) and (36). Let us give some $f_{N, N}^{(6)}$ as a series to show the magnitude of the numerical coefficients involved. The series expansion of $f_{N, N}^{(6)}$ for the first values of $N$ reads:

$$
\begin{array}{ll}
N=0: & \frac{t^{9}}{1073741824}+\frac{37 t^{10}}{8589934592}+\cdots \\
N=1: & \frac{7 t^{12}}{4398046511104}+\frac{21 t^{13}}{2199023255552}+\cdots \\
N=2: & \frac{21 t^{15}}{1125899906842624}+\frac{19215 t^{16}}{144115188075855872}+\cdots \\
N=3: & \frac{10395 t^{18}}{18446744073709551616}+\frac{84315 t^{19}}{18446744073709551616}+\cdots \\
N=4: & \frac{2335905 t^{21}}{75557863725914323419136}+\frac{166783617 t^{22}}{604462909807314587353088}+\cdots
\end{array}
$$

Appendix C.6. $f_{1,1}^{(7)}, f_{1,1}^{(8)}$ and $f_{1,1}^{(9)}$

Here we give the $f_{N, N}^{(j)}$ for the other values of $j=7,8,9$ and $N=1$ :

$$
\begin{aligned}
5040 t^{1 / 2} f_{1,1}^{(7)}=2304 \cdot(K-E) \\
\quad-784(2 t-3) \cdot K^{3}-4704 K^{2} E+2352 K E^{2} \\
\quad+840(2 t-3) K^{4} E-840 K^{2} E^{3} \\
\quad+56(4 t-5)(2 t-3) K^{5}+2520 K^{3} E^{2} \\
\quad-28(4 t-5)(2 t-3) E K^{6}-210(2 t-3) E^{2} K^{5} \\
\quad-\left(32 t^{3}-156 t^{2}+228 t-105\right) K^{7}+105 K^{3} E^{4}-420 K^{4} E^{3} \\
645120 f_{1,1}^{(8)}=11025-38748 K E-12916(t-2) K^{2} \\
\quad+29610 K^{2} E^{2}+1974\left(t^{2}-6 t+6\right) K^{4}+19740(t-2) K^{3} E \\
\quad-8820 K^{3} E^{3}-84(t-2)\left(t^{2}-10 t+10\right) K^{6} \\
\quad-1764\left(t^{2}-6 t+6\right) K^{5} E-8820(t-2) K^{4} E^{2} \\
\quad+945 K^{4} E^{4}+\left(t^{4}-20 t^{3}+48 t^{2}-56 t+28\right) K^{8} \\
\quad+36(t-2)\left(t^{2}-10 t+10\right) K^{7} E+378\left(t^{2}-6 t+6\right) K^{6} E^{2} \\
\quad+1260(t-2) K^{5} E^{3}
\end{aligned}
$$




$$
\begin{aligned}
& 362800 t^{1 / 2} f_{1,1}^{(9)}=147456 \cdot(K-E) \\
& \quad+157440 K^{2}-52480(2 t-3) \cdot K^{3}-314880 K^{2} E \\
& \quad-65520 K^{2} E^{3}+65520(2 t-3) K^{4} E \\
& \quad+4368(4 t-5)(2 t-3) K^{5}+196560 K^{3} E^{2} \\
& \quad+12600 K^{3} E^{4}-50400 K^{4} E^{3}-3360(4 t-5)(2 t-3) K^{6} E \\
& \quad+120\left(105-228 t+156 t^{2}-32 t^{3}\right) K^{7} \\
& \quad-25200(2 t-3) K^{5} E^{2}+630(4 t-5)(2 t-3) \cdot K^{7} E^{2} \\
& \quad-945 E^{5} K^{4}+4725 E^{4} K^{5}+3150(2 t-3) K^{6} E^{3} \\
& \quad+45\left(32 t^{3}-156 t^{2}+228 t-105\right) K^{8} E \\
& \quad+\left(128 t^{4}-960 t^{3}+2460 t^{2}-2572 t+945\right) K^{9}
\end{aligned}
$$

\section{Appendix D. Miscellaneous off diagonal $j$-particle contributions}

We display here some off diagonal $j$-particle contributions.

$$
\begin{aligned}
2 s^{2} & \cdot C^{(2)}(0,2)=2 s^{2}-2\left(1+s^{2}\right) s^{2} \cdot K \\
& +\left(2 s^{4}+s^{2}-2\right) \cdot K^{2}-(s-2)(s+2) K E-2 E^{2} \\
8 & s^{4} \cdot C^{(2)}(0,3)=s^{2}\left(8+27 s^{2}+8 s^{4}\right) \\
& -24 s^{2}\left(1+s^{2}\right) \cdot E-2 s^{2}\left(1+s^{2}\right)\left(-4+13 s^{2}+8 s^{4}\right) \cdot K \\
& +\left(1+s^{2}\right)\left(8 s^{8}+7 s^{6}+3 s^{4}-8 s^{2}-8\right) \cdot K^{2} \\
& +4\left(4+6 s^{2}+7 s^{4}+6 s^{6}\right) \cdot E K-8\left(s^{4}+1\right) \cdot E^{2} \\
18 & s^{6} \cdot C^{(2)}(0,4)=36\left(2+8 s^{2}+13 s^{4}+8 s^{6}+2 s^{8}\right) s^{2} \\
& -24 s^{2}\left(1+s^{2}\right)\left(6 s^{8}+18 s^{6}+11 s^{4}-12 s^{2}-8\right) K \\
& -48 s^{2}\left(1+s^{2}\right)\left(7 s^{4}+15 s^{2}+7\right) E \\
& +\left(s^{2}+2\right)\left(72 s^{12}+144 s^{10}-60 s^{8}-200 s^{6}+s^{4}+62 s^{2}-16\right) K^{2} \\
& +\left(64-408 s^{2}-576 s^{4}+591 s^{6}+1088 s^{8}+336 s^{10}\right) E K \\
& -4\left(8-93 s^{2}-200 s^{4}-93 s^{6}+8 s^{8}\right) E^{2} \\
8 & s^{2} \cdot C^{(2)}(1,2)=7 s^{2}-4\left(1+s^{2}\right) \cdot E \\
& -2\left(1+s^{2}\right)\left(s^{2}-2\right) \cdot K \\
& +\left(1+s^{2}\right)\left(4+3 s^{2}-5 s^{4}\right) K^{2} \\
& +4\left(s^{4}-3 s^{2}-3\right) E K+8 E^{2} \\
6 & s^{4} \cdot C^{(2)}(1,3)=9 s^{4}-4\left(1+s^{2}\right)\left(s^{4}+3 s^{2}+1\right) \cdot E \\
& +4\left(1+s^{2}\right)\left(1+3 s^{2}-s^{4}\right) \cdot K \\
& +\left(10+8 s^{2}-2 s^{4}-8 s^{6}-5 s^{8}\right) \cdot K^{2} \\
& +\left(-24-32 s^{2}-13 s^{4}+16 s^{6}+4 s^{8}\right) \cdot E K \\
& +2\left(7 s^{4}+12 s^{2}+7\right) \cdot E^{2} \\
& (10
\end{aligned}
$$




\section{Appendix E. Differential operators in the scaling limit}

The differential operators $L_{6}^{\text {scal }}, \cdots, L_{10}^{\text {scal }}$ introduced in sec. 5 read:

$$
\begin{aligned}
& L_{6}^{\text {scal }}=64 x^{6} D x^{6}+320 x^{5} D x^{5}-16 x^{4}\left(48+35 x^{2}\right) D x^{4} \\
& +32 x^{3}\left(91 x^{2}-4\right) D x^{3}+4 x^{2}\left(848-1788 x^{2}+259 x^{4}\right) D x^{2} \\
& -20 x\left(80-380 x^{2}+383 x^{4}\right) D x \\
& -225 x^{6}-2480 x^{2}+1600+17580 x^{4} \\
& L_{7}^{\text {scal }}=4 x^{7} D x^{7}-56\left(3+x^{2}\right) x^{5} D x^{5}+8\left(41+84 x^{2}\right) x^{4} D x^{4} \\
& +4\left(69-810 x^{2}+49 x^{4}\right) x^{3} D x^{3} \\
& -8\left(251-971 x^{2}+372 x^{4}\right) x^{2} D x^{2} \\
& +4\left(275-2116 x^{2}+4212 x^{4}-36 x^{6}\right) x D x \\
& -1100+2832 x^{2}-35280 x^{4}+1152 x^{6} \text {, } \\
& L_{8}^{\text {scal }}=256 x^{8} D x^{8}-1024 x^{7} D x^{7}-5376\left(2+x^{2}\right) x^{6} D x^{6} \\
& +256\left(334+399 x^{2}\right) x^{5} D x^{5} \\
& -32\left(4040+26304 x^{2}-987 x^{4}\right) x^{4} D x^{4} \\
& -64\left(4216-57384 x^{2}+12027 x^{4}\right) x^{3} D x^{3} \\
& +16\left(76688-537424 x^{2}+482478 x^{4}-3229 x^{6}\right) x^{2} D x^{2} \\
& -16\left(48400-598032 x^{2}+2328262 x^{4}-60013 x^{6}\right) x D x \\
& +774400-3342592 x^{2}+72498272 x^{4}-4879248 x^{6}+11025 x^{8} \text {, } \\
& L_{9}^{\text {scal }}=4 x^{9} D x^{9}+480 x^{8} D x^{8}-24\left(5 x^{2}-961\right) x^{7} D x^{7} \\
& +8\left(71315-1092 x^{2}\right) x^{6} D x^{6} \\
& +12\left(645013-19478 x^{2}+91 x^{4}\right) x^{5} D x^{5} \\
& +8\left(6985303+4920 x^{4}-354291 x^{2}\right) x^{4} D x^{4} \\
& +4\left(44460417-3774790 x^{2}+108828 x^{4}-820 x^{6}\right) x^{3} D x^{3} \\
& -4\left(443021+5872124 x^{2}-382676 x^{4}+9216 x^{6}\right) x^{2} D x^{2} \\
& +4\left(576 x^{8}-16128 x^{6}+94812 x^{4}+9265148 x^{2}-268975475\right) x D x \\
& +1024\left(36 x^{6}-2019 x^{4}+66804 x^{2}-1254400\right) \\
& L_{10}^{\text {scal }}=1024 x^{10} D x^{10}+168960 x^{9} D x^{9}+8448\left(1368-5 x^{2}\right) x^{8} D x^{8} \\
& +11264\left(37880-399 x^{2}\right) x^{7} D x^{7} \\
& +4224\left(2194904-44164 x^{2}+133 x^{4}\right) x^{6} D x^{6} \\
& +128\left(946138408-30108276 x^{2}+259215 x^{4}\right) x^{5} D x^{5} \\
& +32\left(29025917984-1305848840 x^{2}+21377796 x^{4}-86405 x^{6}\right) x^{4} D x^{4} \\
& +64\left(60403578784-3569603544 x^{2}+92712956 x^{4}-1057221 x^{6}\right) x^{3} D x^{3} \\
& +4\left(1794785734400-134201812672 x^{2}+5056843872 x^{4}\right. \\
& \left.-110074968 x^{6}+1057221 x^{8}\right) x^{2} D x^{2}
\end{aligned}
$$


Holonomy of the Ising model form factors

$$
\begin{aligned}
& +972\left(3056659200-330174912 x^{2}+18778592 x^{4}-694968 x^{6}\right. \\
& \left.+18375 x^{8}\right) \cdot x D x \quad-893025 x^{10}
\end{aligned}
$$

[1] L. Onsager, Crystal Statistics, A two dimensional model with an order disorder transition, Phys. Rev. 65 (1944) 117

[2] B. Kaufman, Crystal Statistics II. Partition function evaluated by spinor analysis, Phys. Rev. 76 (1949) 1232

[3] L. Onsager, Nuovo Cimento 6 Suppl. (1949) 261

[4] C.N. Yang, The spontaneous magnetization of the two dimensional Ising model, Phys. Rev. 85 (1952) 808

[5] B. Kaufman and L. Onsager, Short-range order in a binary Ising lattice, Phys. Rev. 76 (1949) 1244

[6] E.W. Montroll, R.B. Potts and J.C. Ward, Correlations and spontaneous magnetization of the two-dimensional Ising model, J. Math. Phys. 4 (1963) 308

[7] T.T. Wu, Theory of Toeplitz determinants and the spin correlations of the two-dimensional Ising model, Phys. Rev. 149 (1966) 380

[8] H.Cheng and T.T. Wu, Theory of Toeplitz determinants and the spin correlations of the twodimensional Ising model III, Phys. Rev. 164 (1967) 719-735

[9] T.T. Wu, B.M. McCoy, C.A. Tracy and E. Barouch, 1976 Phys. Rev. B 13, 316-374

[10] B. Nickel, On the singularity structure of the Ising model susceptibility, J. Phys. A 32 (1999) 3889

[11] B. Nickel, Addendum to 'On the singularity structure of the Ising model susceptibility', J. Phys. A 33 (2000) 1693

[12] D. Hansel, J.M; Maillard, J. Oitmaa and M.J. Vergakis, J. Stat. Phys. 48 (1987)69-80

[13] A.J. Guttmann and I. G. Enting, Solvability of Some Statistical Mechanical Sytems, Phys. Rev. Lett. 76, (1966) 344-347

[14] W.P. Orrick, B.G. Nickel, A.J. Guttmann, J.H.H. Perk, (2001) J. Stat. Phys. 102 795-841

[15] N. Zenine, S. Boukraa, S. Hassani, J.M. Maillard, J. Phys. A: Math. Gen. 37 (2004)9651-9668 and arXiv:math-ph/0407060

[16] N. Zenine, S. Boukraa, S. Hassani, J.M. Maillard, J. Phys. A: Math. Gen. 38(2005) 1875-1899 and arXiv:hep-ph/0411051

[17] N. Zenine, S. Boukraa, S. Hassani, J.M. Maillard, J. Phys. A: Math. Gen. 38 4149-4173 (2005) and arXiv: cond-mat/0502155

[18] N. Zenine, S. Boukraa, S. Hassani and J-M. Maillard, J. Phys. A 38 (2005) 9439-9474 and arXiv:hep-th/0506214

[19] S.Boukraa, S.Hassani, J.M.Maillard, B.M. McCoy, J-A. Weil and N.Zenine, Painlevé versus Fuchs, Special issue on Painleve VI, J. Phys. A 39 (2006) 12245-12263, and arXiv: math-ph 0602010

[20] M. Jimbo and T. Miwa, Studies on holonomic quantum fields. XVII, Proc. Japan. Acad. 56A (1980) 405-410; 57A (1981) 347

[21] B. McCoy, C.A. Tracy and T.T. Wu, Painleve equations of the third kind, J. Math. Phys. 18 (1977) 1058

[22] M.F. Singer, Testing reducibility of linear differential operators: A group theoretic perspective., 1996, Appl. Alg. Eng. Commun. Comp. 7, no2, 77-104

[23] M. van Hoeij, Rational Solutions of the Mixed Differential Equation and its Application to Factorization of Differential Operators, in: Proceedings ISSAC '96, 219-225, ACM, NewYork

[24] M. van der Put and M.F Singer, Galois theory of linear differential equations. Grundlehren der Mathematischen Wissenschaften, 328. Springer-Verlag, Berlin, (2003)

[25] S.Cecotti and C.Vafa, Ising model and $n=2$ supersymmetric theories, Comm. Math. Phys. 157 (1993) 139-178

[26] J. Palmer and C.A. Tracy, Two dimensional Ising correlation functions: convergence in the scaling limit, Adv. in Appl. Math. 2 (1981) 329

[27] K. Yamada, On the spin-spin correlation function of the Ising square lattice and the zero field susceptibility, Progr. Theor. Phys. 71 (1984) 1416-1418 
[28] J. Stephenson, Ising model spin correlations on triangular lattices, J. Math. Phys. 5 (1964) 1009

[29] B.M. McCoy and T.T. Wu, Nonlinear partial difference equations for the two-dimensional Ising model, Phys. Rev. Lett. 45 (1980) 675

[30] Erdeleyi, Bateman manuscript project, higher transcendental functions; McGraw Hill, N-Y, (1955)

[31] R.K. Ghosh, On the low temperature series expansion for the diagonal correlation functions in the two-dimensional Ising model, arXiv: cond-mat/0505166

[32] Yu. I. Manin, Sixth Painlevé equation, Universal elliptic curve, and mirror of $P^{2}$, AMS Transl. (2) vol. 186 (1998) 131-151

[33] D. Guzzetti, The Elliptic Representation of the General Painlevé 6 Equation, and arXiv: math.CV/0108073 v2 2001

[34] V. V Bazhanov, V. V Mangazeev, The eight-vertex model and Painleve VI, Special issue on Painlevé VI, J. Phys. A 39 (2006) 12235-12243, and arXiv: hep-th 0602122

[35] M. Murata, H. Sakai and J. Yoneda, Riccati solutions of discrete Painlevé equations with Weyl group symmetry of type $E_{8}^{(1)}$, J. Math. Phys. 44, (2003) 1396-1414

[36] H. Sakai, Rational Surfaces Associated with Affine Root Systems and Geometry of the Painlevé Equations, Comm. Math. Phys. 220, (2001) 165-229.

[37] W. Barth and J. Michel, Modular curves and Poncelet polygons, Math. Ann. 295 (1993), 25-49. MR 94c:14045

[38] P. Boalch, The fifty-two icosahedral solutions to Painlevé VI, math.AG/0406281

[39] P.Boalch, Some explicit solutions to the Riemann-Hilbert problem, math.DG/0501464

[40] N.J. Hitchin, Poncelet Polygons and the Painlevé equations, in Geometry and analysis (Bombay, 1992) 151-185, Tata Inst. Fund. Res., Bombay (1995)

[41] E. Picard, Mémoire sur la theorie des functions algébriques de deux variables, Journal de Liouville, 5 (1889) 135-319

[42] M. Mazzocco, Picard and Chazy solutions to the Painlevé VI equation, Math. Ann. 321 (2001) 157-195, and arXiv: math.AG/9901054

[43] B. Ben Hamed and L. Gavrilov, Int. Math. Res. Notices, No. 60 (2005) 3728

[44] K. Okamoto, Ann. Mat. Pura Appl. 146 (1987) 337-381

[45] R.K. Ghosh and R.E. Shrock, Exact expressions for diagonal correlation functions in the $d=2$ Ising model, Phys. Rev. B 30,(1984) 3790-3794

[46] H.E. Boos and V.E. Korepin, Quantum spin chains and Riemann zeta function with odd arguments, J. Phys. A 34 (2001) 5311-5316

[47] H.E. Boos and V.E. Korepin, Evaluation of integrals representing correlations in the XXX Heisenberg spin chain, in MathPhys Odyssey 2001 eds. M. Kashiwara and T. Miwa (Birkhäuser, 2002) 65-108

[48] H. E. Boos, F. Göhmann, A. Klümper and J. Suzuki, The 75th Anniversary of the Bethe Ansatz, Factorization of multiple integrals representing the density matrix of a finite segment of the Heisenberg spin chain, J. Stat. Mech. (2006) P04001

[49] D. Kreimer, Knots and Feynman Diagrams, Cambridge Lecture Notes in Physics 13, Cambridge University Press (2000), Chapter 9

[50] D.H. Bailey, J.M. Borwein and R.E. Crandall, Integrals of the Ising class, (2006) J. Phys. A 39, 12271-12302 